Supporting Information

\title{
Ion Dissociation in Ionic Liquids and Ionic Liquid Solutions
}

Oscar Nordness and Joan F. Brennecke*

McKetta Department of Chemical Engineering, University of Texas at Austin, Austin, Texas 78712, United States

*Email: jfb@che.utexas.edu 


\section{Organization and Structure of Supporting Information:}

We begin by outlining the supporting information associated with this review paper. It should be noted that a great deal of the information used in this work is provided in excel files for ease of access and convenience to the reader, including the references for all the data used to calculate ion dissociations. Additionally, we outline the framework used for calculating the ion dissociations of both neat ILs and ILsolvent systems, as well as the VFT equations used to fit changes in neat viscosity and ionic conductivity data with temperature.

This document contains the following Tables and Figures:

\section{Supporting Information Tables:}

Table S1a. Cation names, abbreviations and 2D structures

Table S1b. Anion names, abbreviations and 2D structures

Table S1c. Solvent names, abbreviations, and 2D structures

Table S2a. UNIFAC $\boldsymbol{r}$ and $\boldsymbol{q}$ parameters and calculated Stokes radii $\left(\boldsymbol{s}^{+}\right)$for the cations in this work, as well as the UNIFAC group contribution sources used to calculate these values.

Table S2b. UNIFAC $\boldsymbol{r}$ and $\boldsymbol{q}$ parameters and calculated Stokes radii $\left(\boldsymbol{s}^{-}\right)$for the anions in this work as well as the UNIFAC group contribution sources used to calculate these values.

\section{Supporting Information Figures:}

Figure S1: Flowchart of ion dissociation calculations and data analysis for neat IL systems

Figure S2: Flowchart of ion dissociation calculations and data analysis for IL/solvent systems.

Figure S3: Visual representation of region selection for ILThermo data: a. representation of a mixture with no overlapping data and $\mathbf{b}$. representation of the valid $x_{I L}$ range for ion dissociation calculations

Figure S4: Interpolated densities and viscosities of the $\left[\mathrm{C}_{4} \operatorname{mpyrr}\right]\left[\mathrm{Tf}_{2} \mathrm{~N}\right]+$ acetonitrile system; the interpolated values are used to calculate the ion dissociation values

We also provide two Excel files. The information in the Supporting Excel Files is as follows:

\begin{tabular}{|l|l|}
\hline \multicolumn{2}{|c|}{ Neat_IL_Supporting_Info.xlsx } \\
\hline Sheet Name & \multicolumn{1}{c|}{ Content } \\
\hline Dissociation & IL Property Values at 298 K for ILs: \\
& $\bullet$ Ion dissociation \\
& $\bullet$ Viscosity \\
& $\bullet$ Ionic conductivity \\
& $\bullet$ Molar conductivity \\
& Tensity \\
& Stokes Radii: s+, s- \\
& IL Molecular Weight (MW) \\
& Reference Numbers \\
\hline
\end{tabular}




\begin{tabular}{|l|l|}
\hline & \\
\hline Conductivity VFT & Conductivity VFT Parameters (A, B, C) \\
& $\begin{array}{l}\mathrm{R}^{2} \text { and AARD of fits } \\
\text { Temperature Range of Conductivity Data } \\
\text { Reference Numbers }\end{array}$ \\
\hline Viscosity VFT & Viscosity VFT Parameters (A, B, C) \\
& Temperature Range of Viscosity Data \\
& $\begin{array}{l}\text { R } 2 \text { and AARD of fits } \\
\text { Reference Numbers }\end{array}$ \\
\hline Density Fit & Density Linear Fit Parameters (A, B) \\
& Temperature Range of Density Data \\
& $\begin{array}{l}\mathrm{R}^{2} \text { and AARD of fits } \\
\text { Reference Numbers }\end{array}$ \\
\hline References & $\begin{array}{l}\text { ILThermo References for neat ILs including paper } \\
\text { title and reference as listed on ILThermo Database }\end{array}$ \\
\hline
\end{tabular}

\begin{tabular}{|l|l|}
\hline \multicolumn{2}{|c|}{ IL_Solvent_Supporting_Info.xlsx } \\
\hline Sheet Name & \multicolumn{1}{c|}{ Content } \\
\hline Binary Sys & $\begin{array}{l}\text { List of IL Solvent Systems from ILThermo } \\
\text { Keys to Binary IL/Solvent System sheets } \\
\text { Range of } x_{I L} \text { values at 298K } \\
\text { Number of datapoints for each system (n) } \\
\text { Reference Numbers } \\
\text { Anion, Cation, and Solvent Abbreviations }\end{array}$ \\
\hline References & $\begin{array}{l}\text { ILThermo References for the IL/Solvent systems } \\
\text { including paper title and reference as listed on } \\
\text { ILThermo Database }\end{array}$ \\
\hline $\begin{array}{l}\text { Data Sheets (Labeled by their assigned key, one } \\
\text { sheet per system) }\end{array}$ & $\begin{array}{l}\text { IL/Solvent Property Values at 298 K for Range } \\
\text { of IL compositions. }\end{array}$ \\
& $\begin{array}{l}\text { - Dissociation } \\
\text { - Viscosity }\end{array}$ \\
\hline
\end{tabular}


Table S1a. Cation names, abbreviations and 2D structures

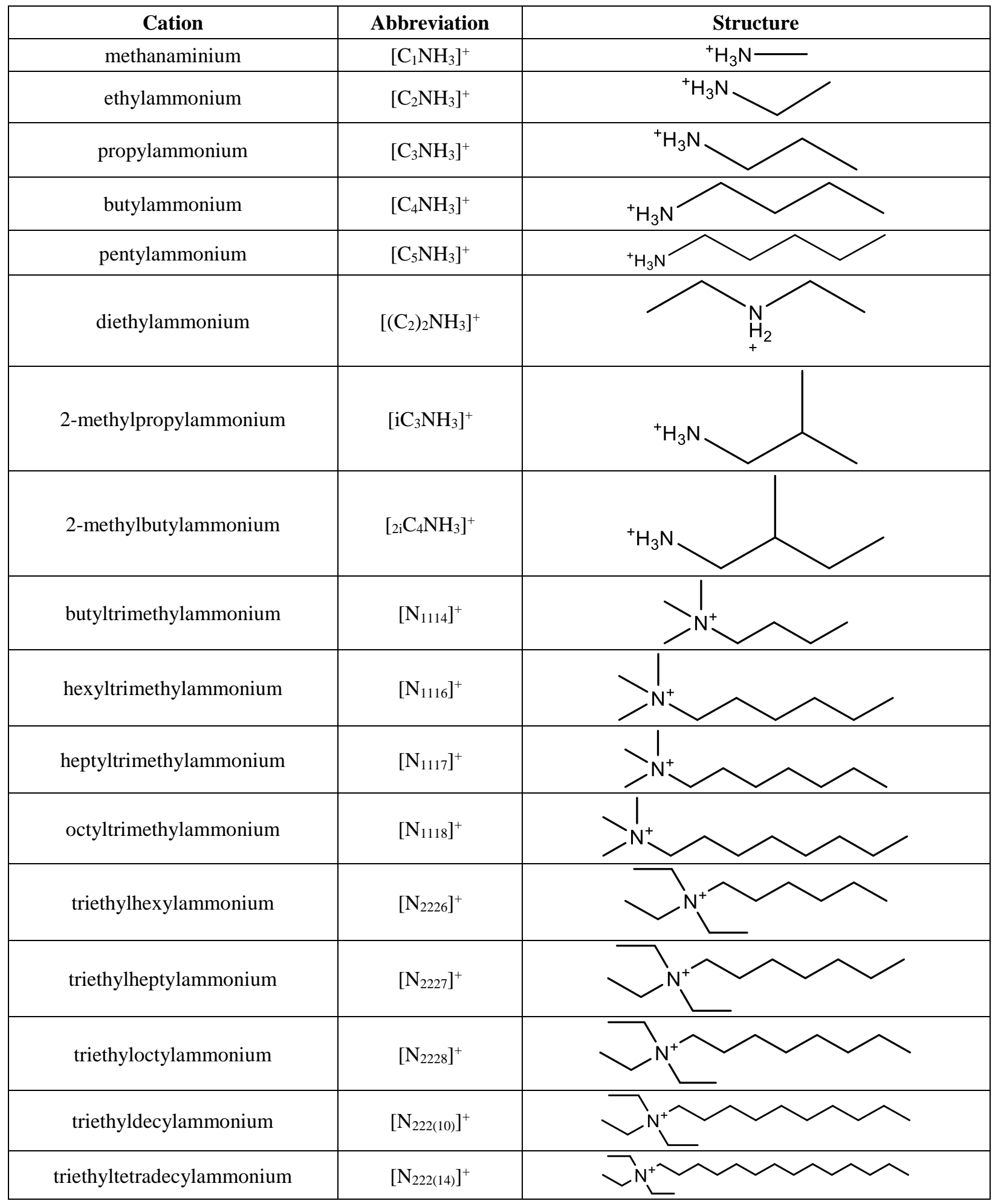




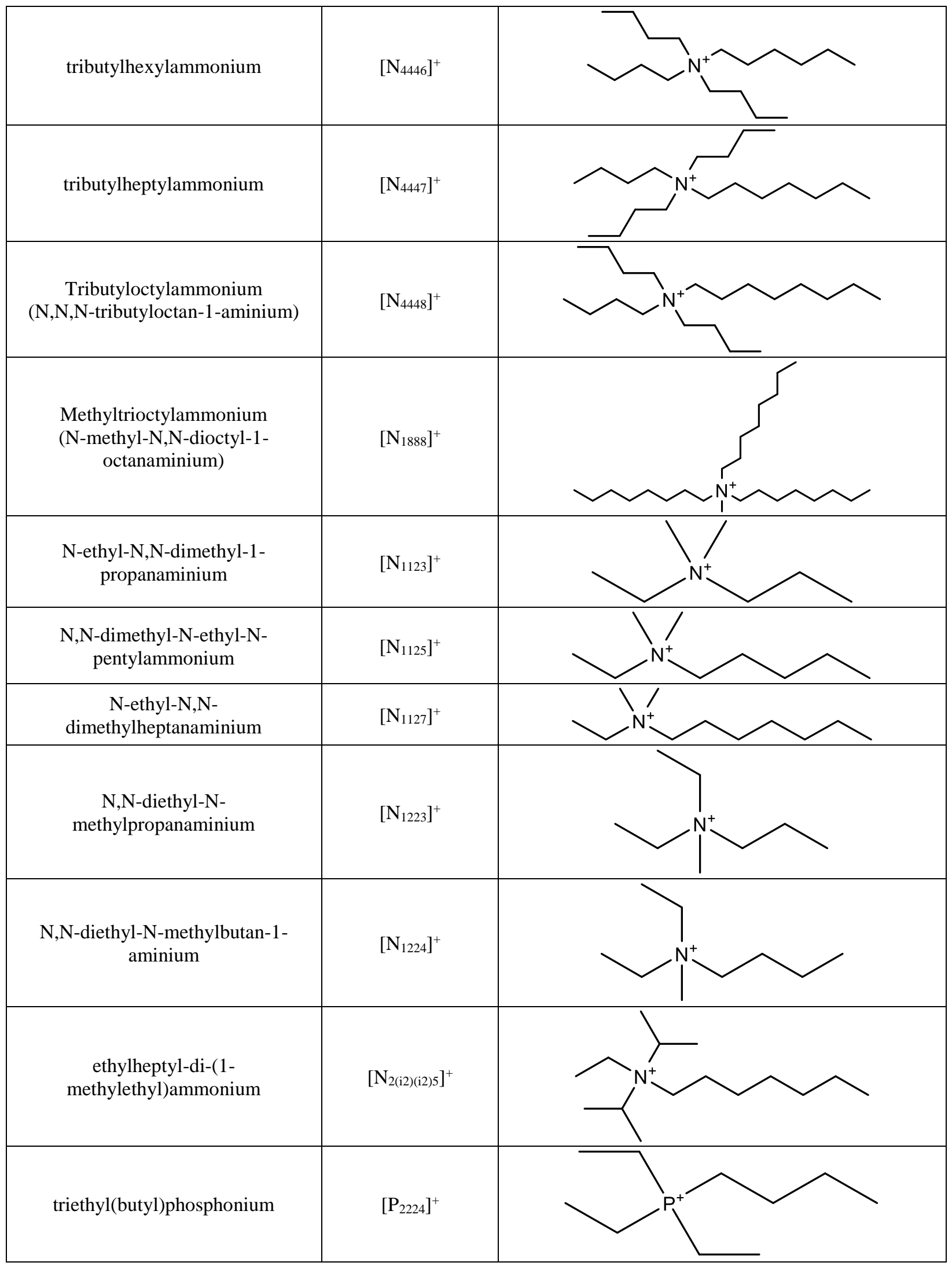




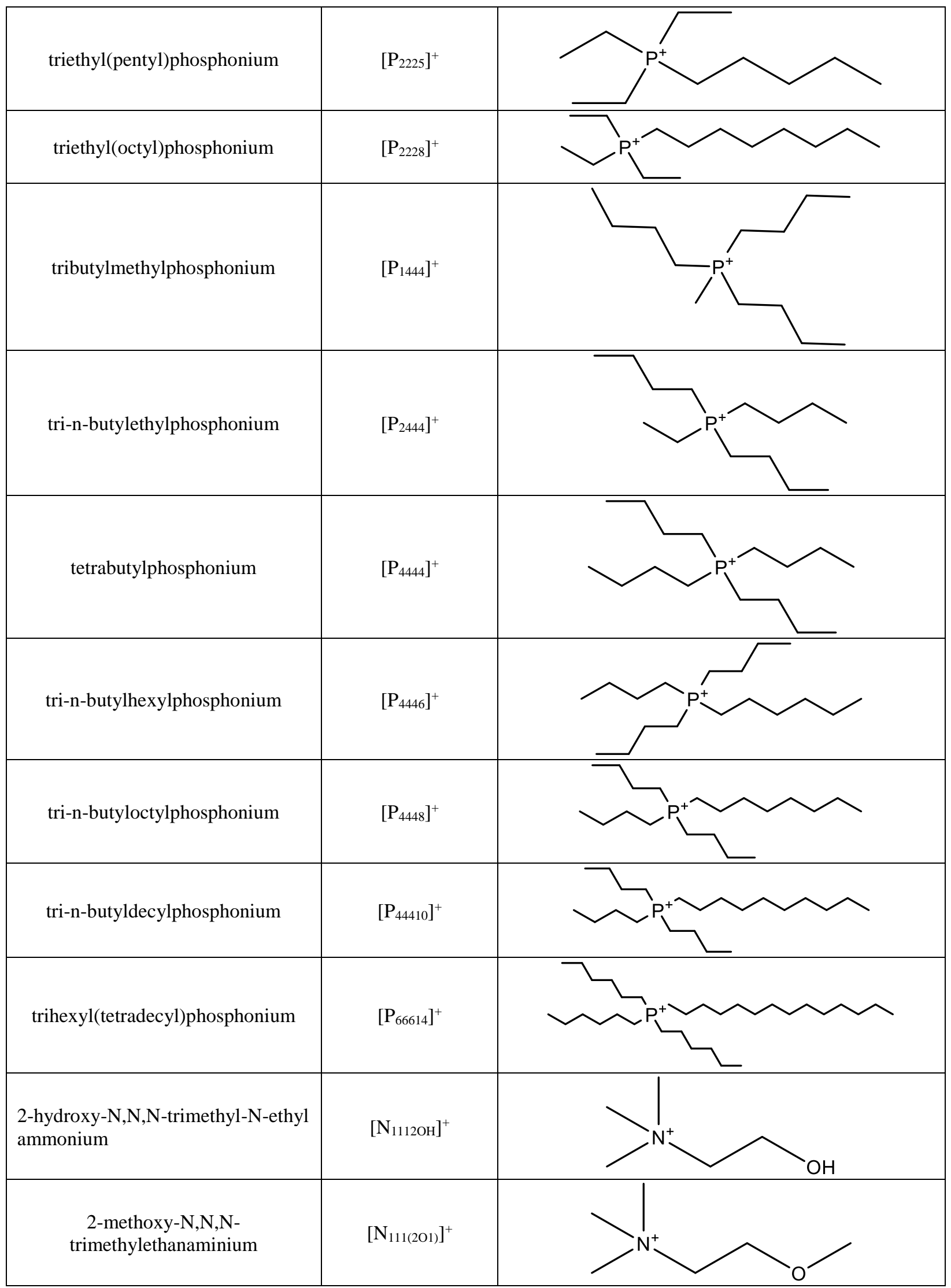




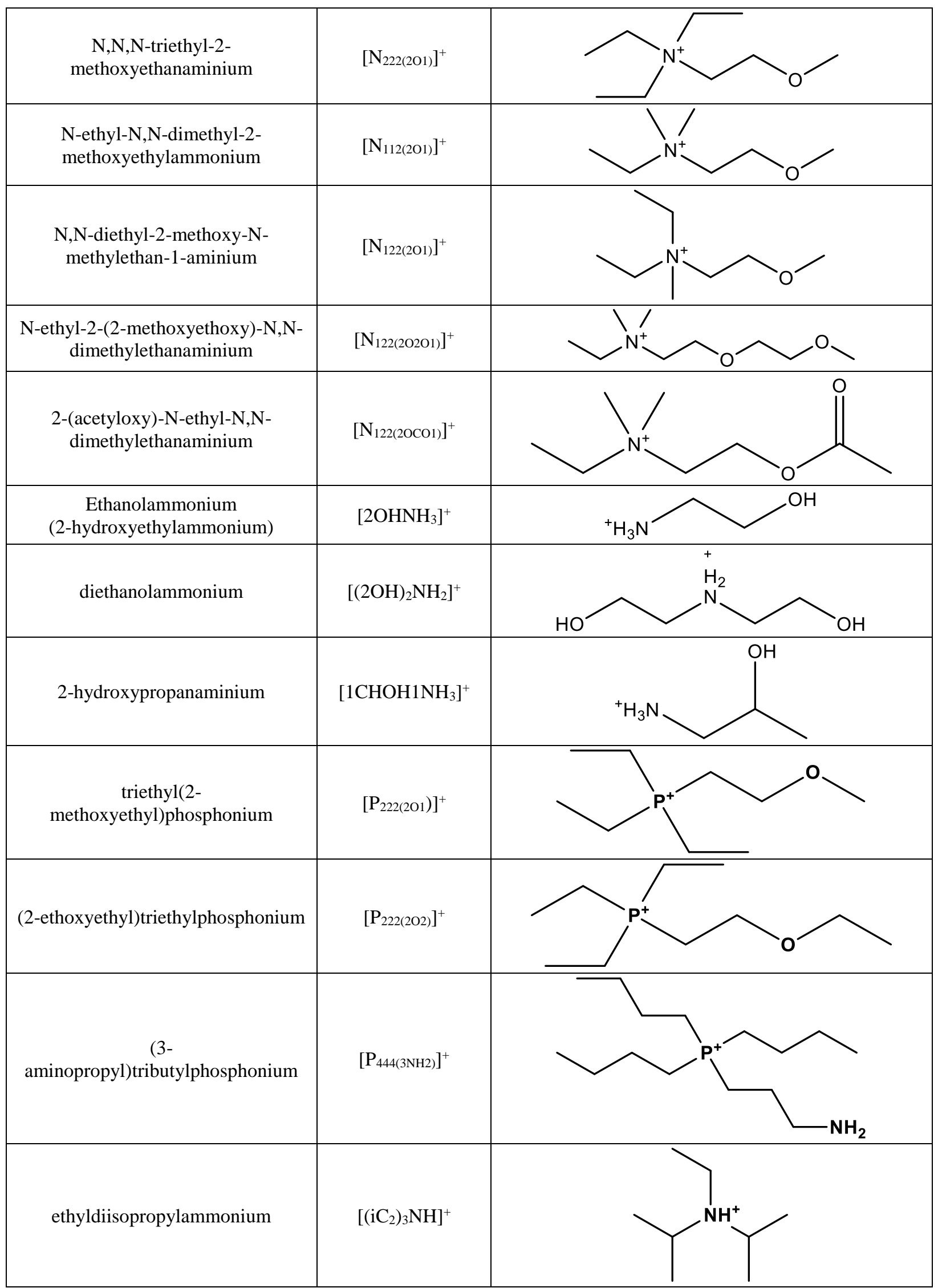




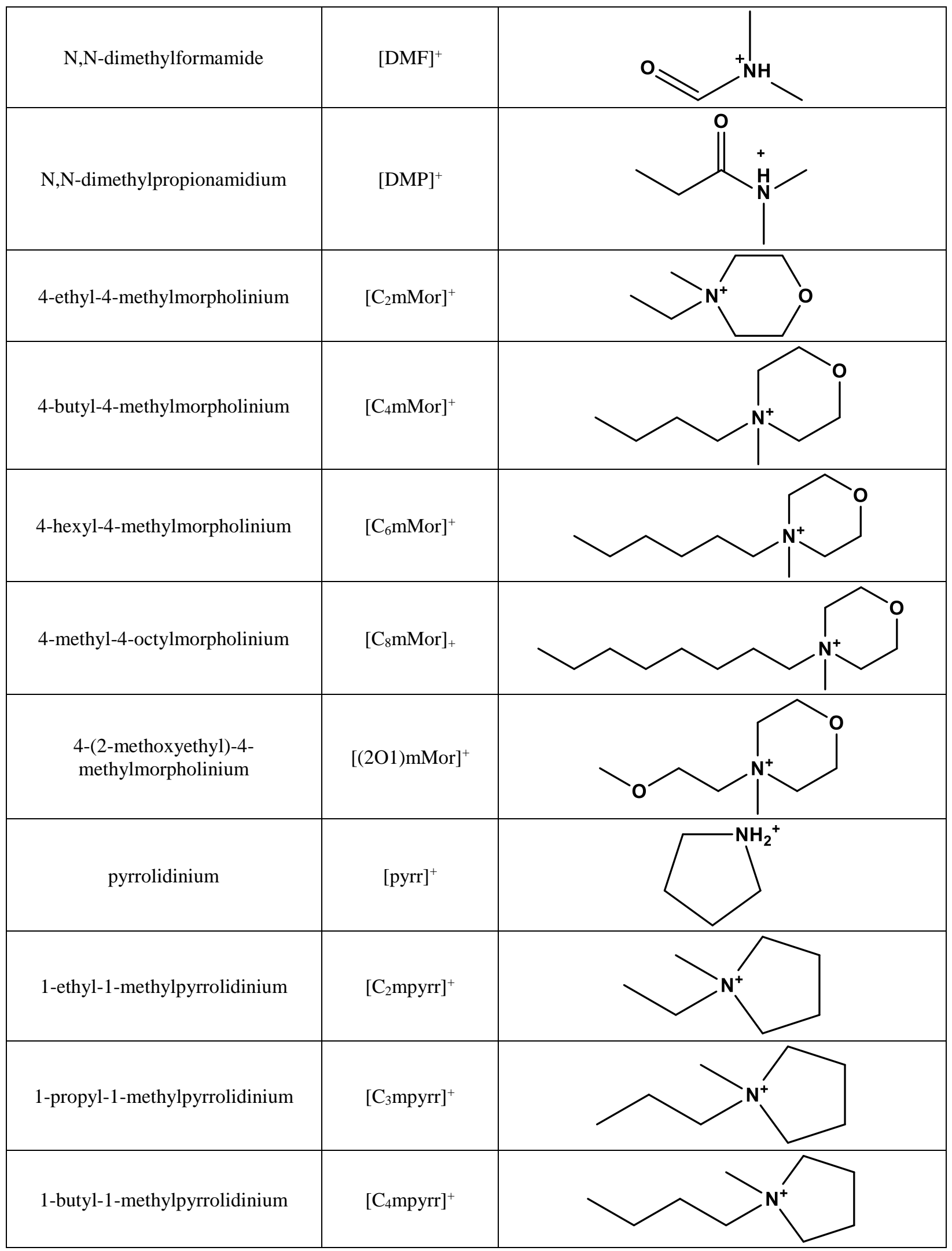




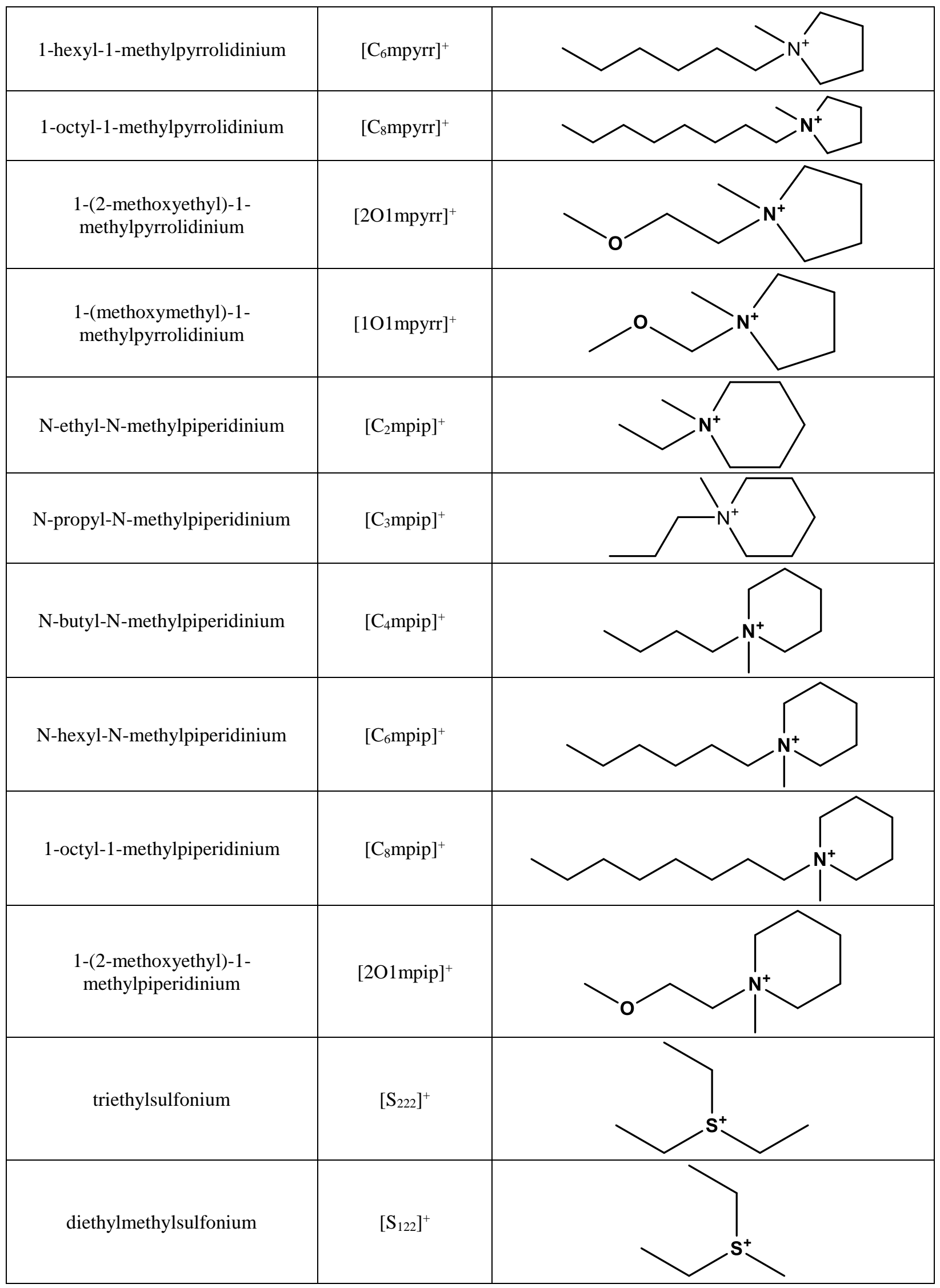




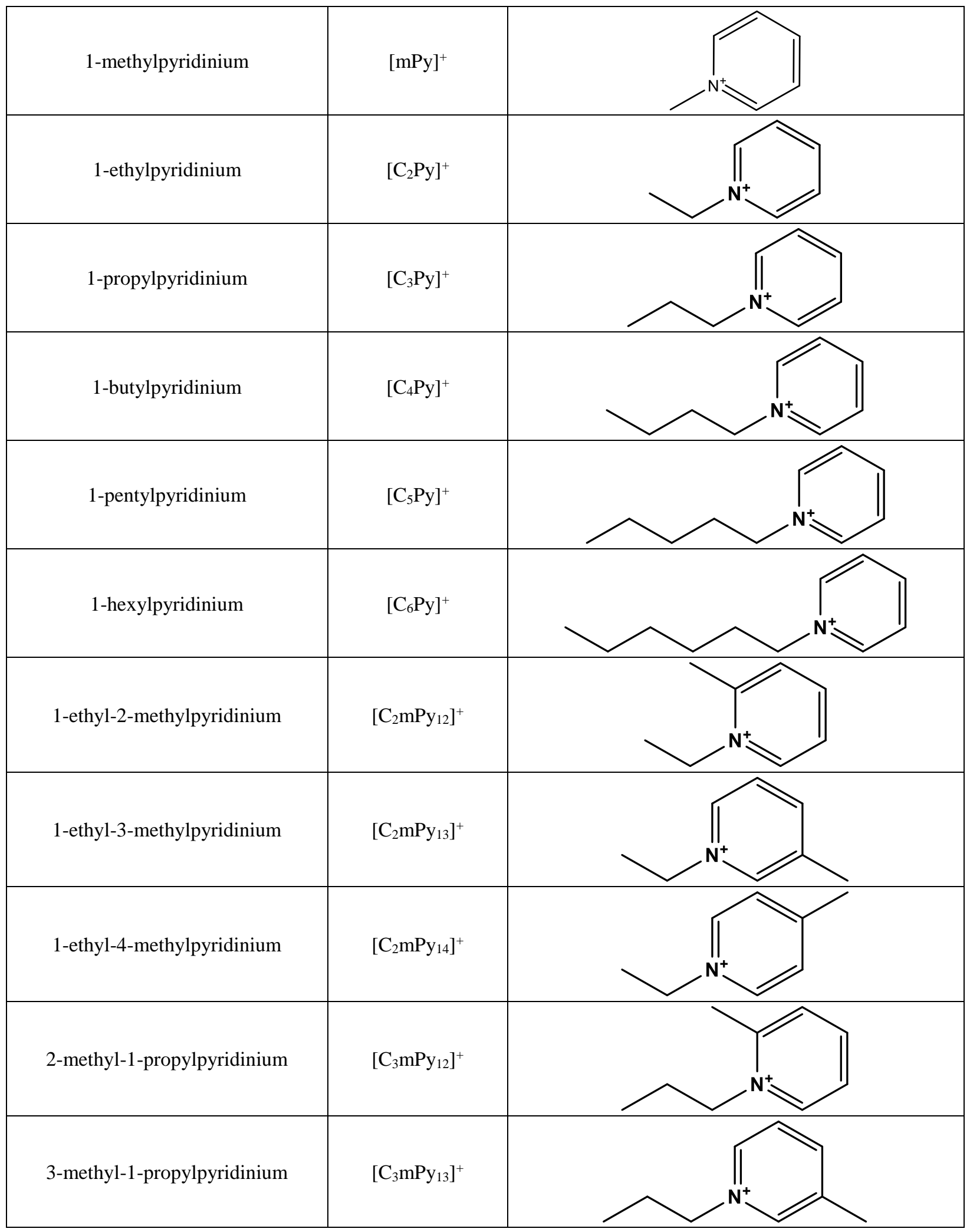




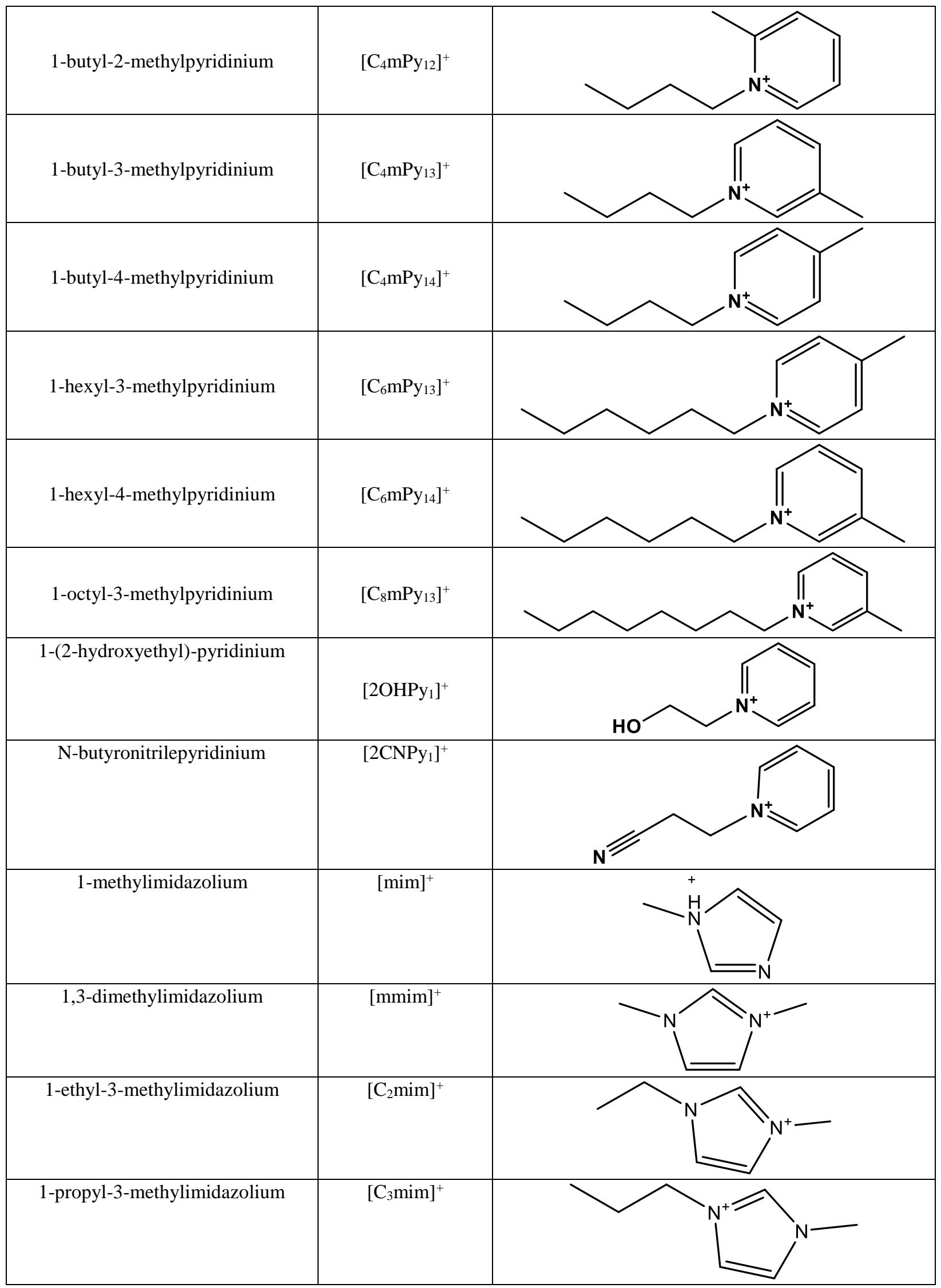




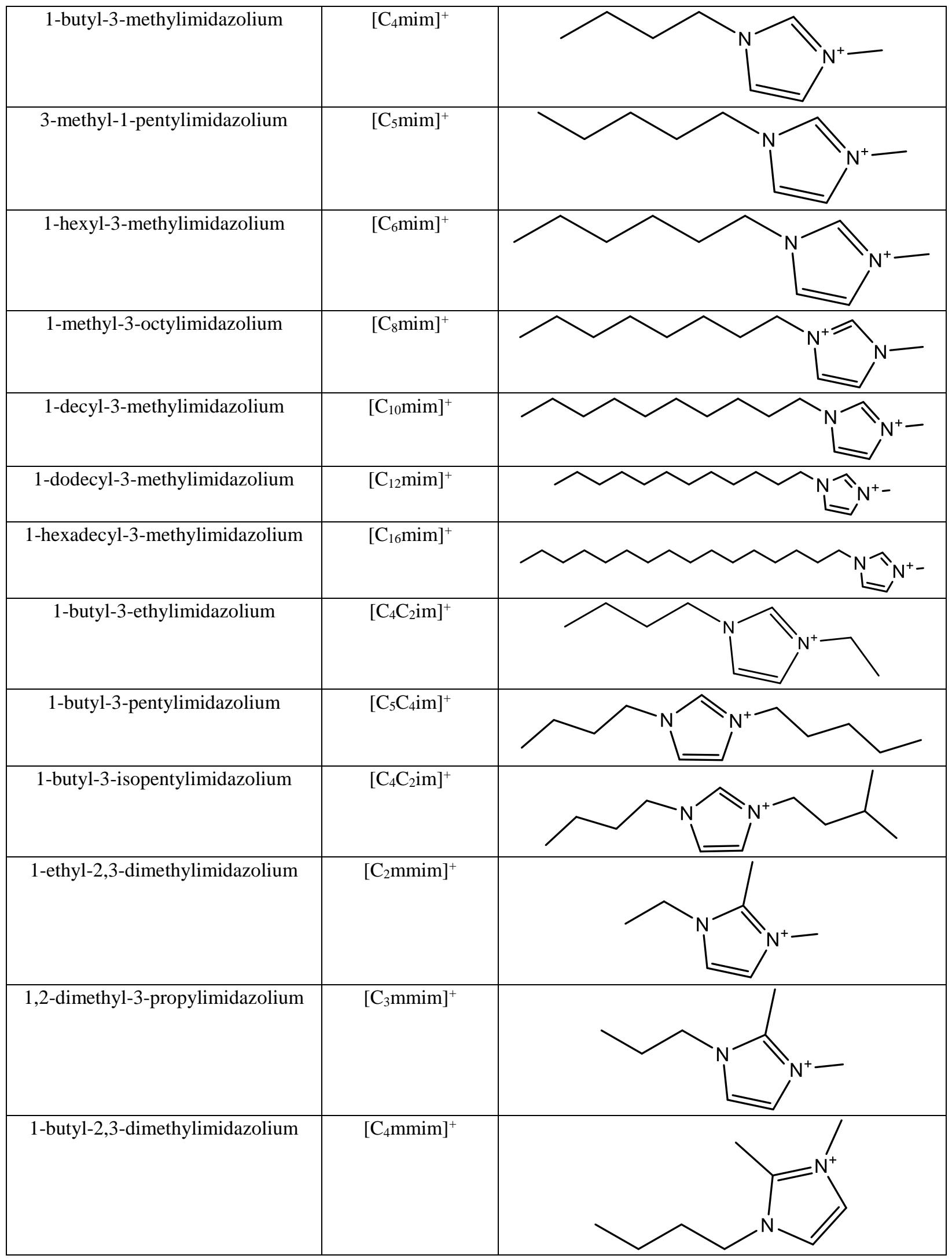




\begin{tabular}{|c|c|c|}
\hline 2,3-dimethyl-1-pentylimidazolium & {$\left[\mathrm{C}_{5} \mathrm{mmim}^{+}\right.$} & \\
\hline 1-hexyl-2,3-dimethylimidazolium & {$\left[\mathrm{C}_{6} \mathrm{mmim}\right]^{+}$} & \\
\hline 1-allyl-3-methylimidazolium & {$[\mathrm{Amim}]^{+}$} & \\
\hline 1-cyclopentyl-3-butylimidazolium & {$\left[\mathrm{cC}_{5} \mathrm{C}_{4} \mathrm{im}\right]^{+}$} & \\
\hline $\begin{array}{l}\text { 1-(1-cyanomethyl)-3- } \\
\text { methylimidazolium }\end{array}$ & {$[1 \mathrm{CNmim}]^{+}$} & \\
\hline $\begin{array}{l}\text { 3-(3-cyanopropyl)-1- } \\
\text { methylimidazolium }\end{array}$ & {$[3 \mathrm{CNmim}]^{+}$} & \\
\hline $\begin{array}{l}\text { 3-(2-(butylamino)-2-oxoethyl)-1- } \\
\text { ethylimidazolium }\end{array}$ & $\underset{+}{\left[(\mathrm{HCONH}) 4 \mathrm{C}_{2} \mathrm{im}\right]}$ & \\
\hline $\begin{array}{l}\text { 1-(2-hydroxyethyl)-3- } \\
\text { methylimidazolium }\end{array}$ & {$[2 \mathrm{OHmim}]^{+}$} & \\
\hline
\end{tabular}


Table S1b. Anion names, abbreviations and 2D structures

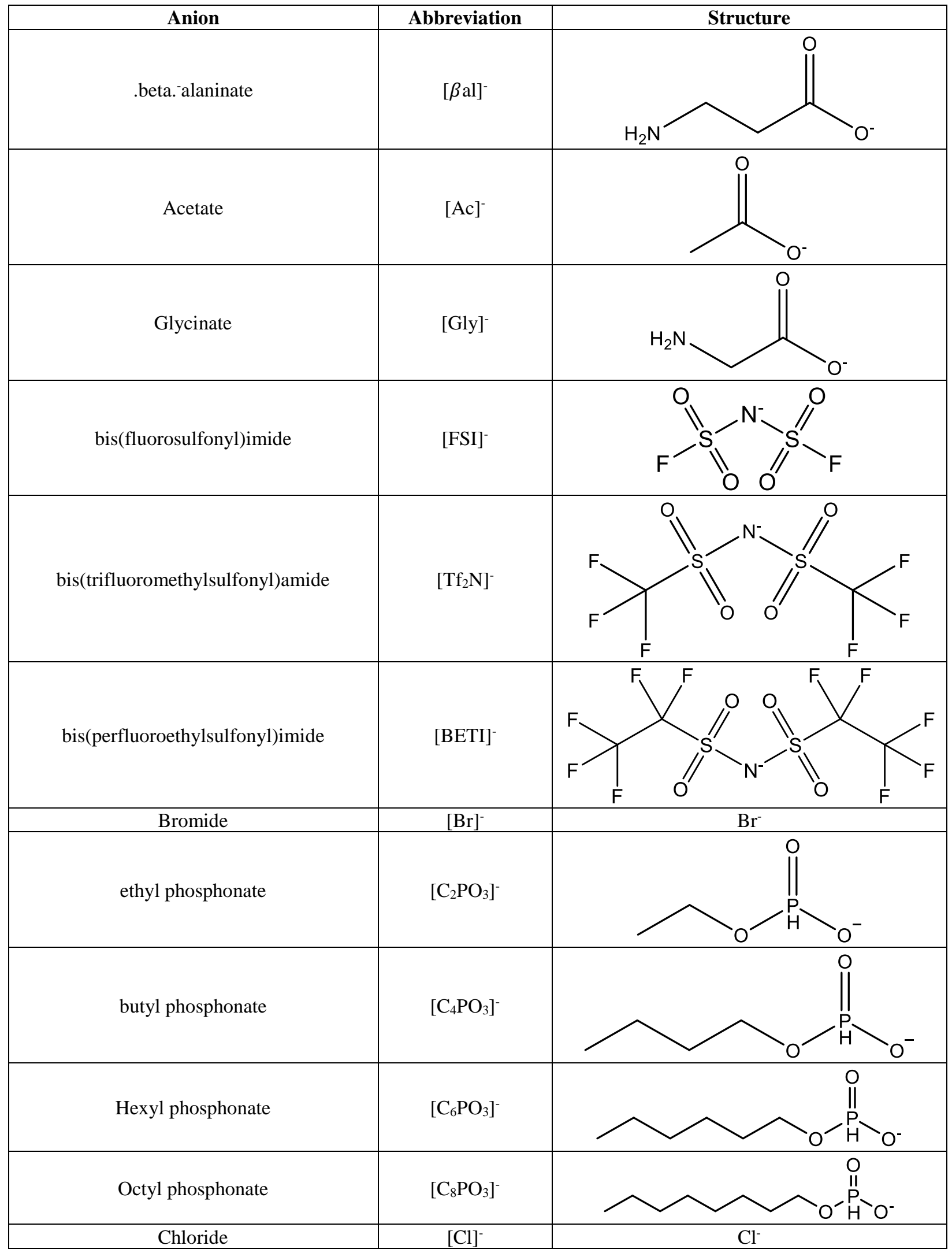




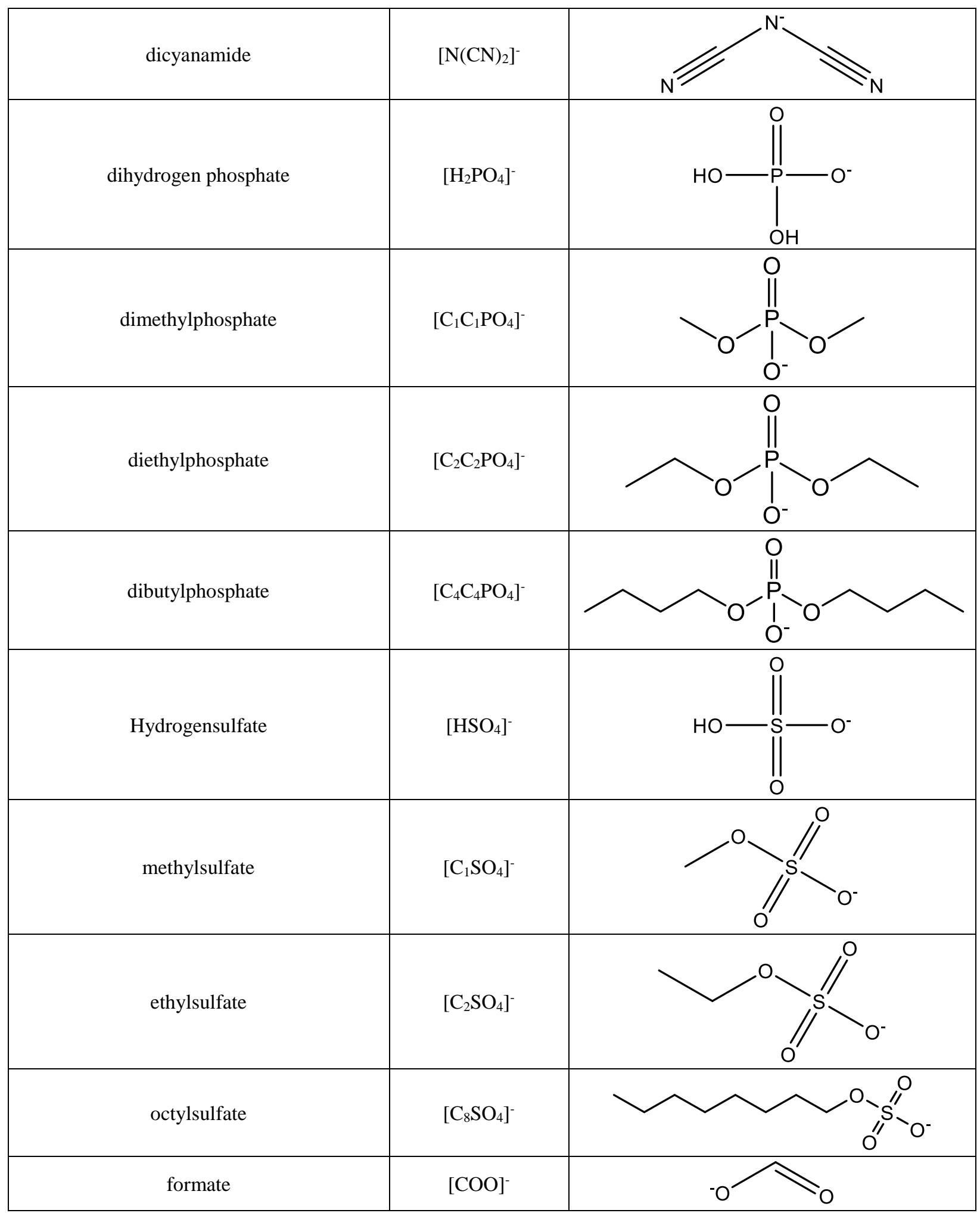




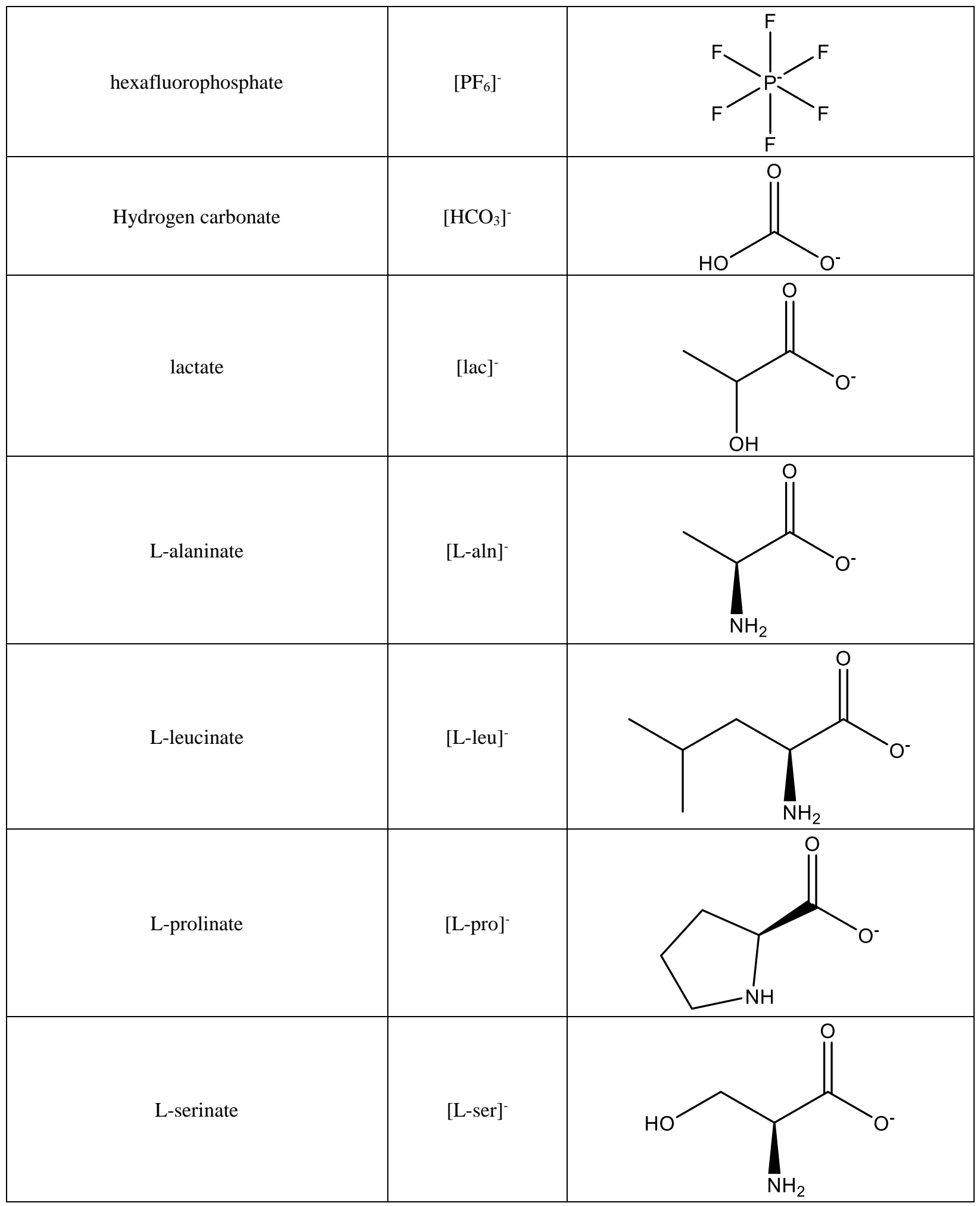




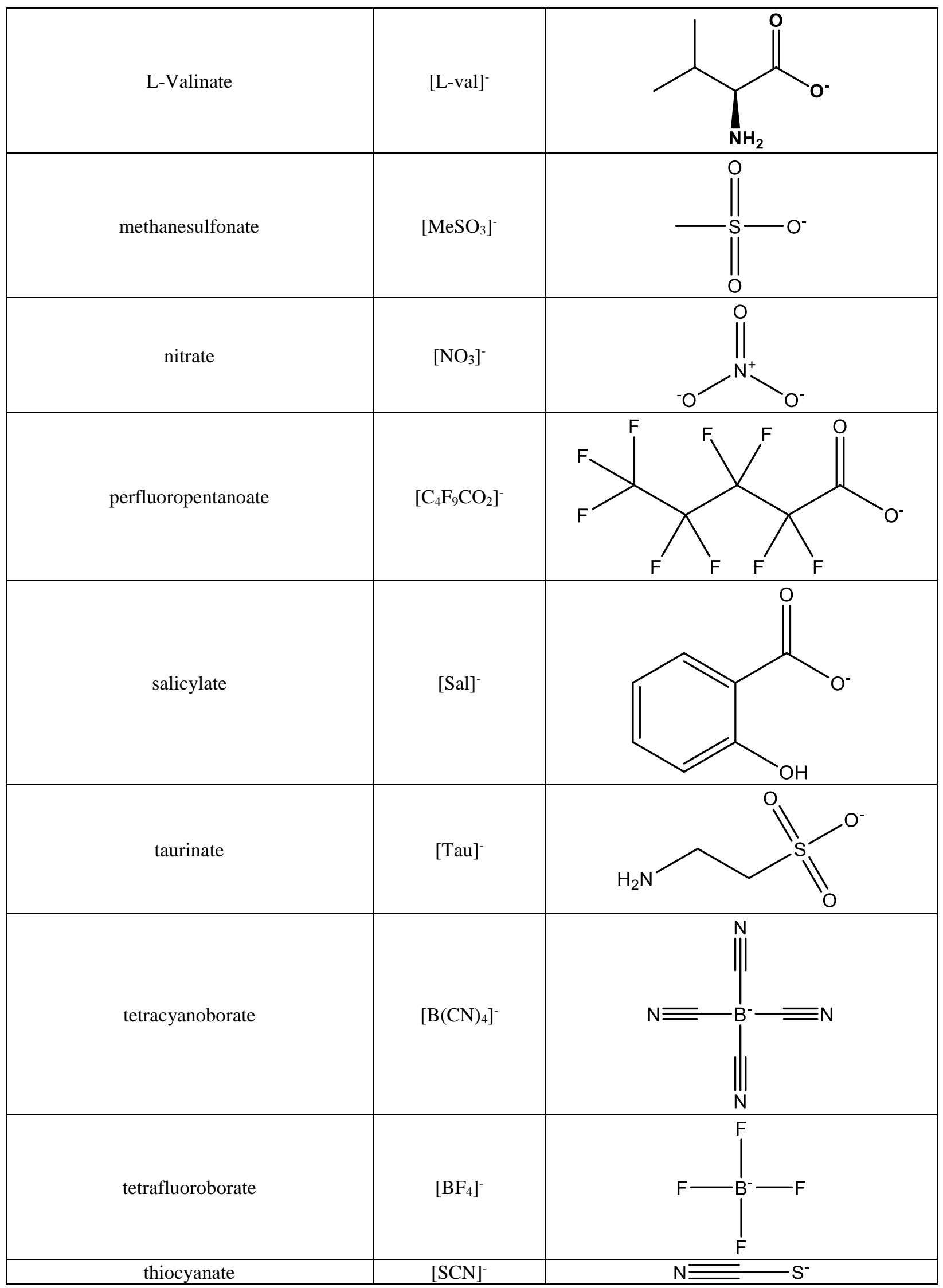




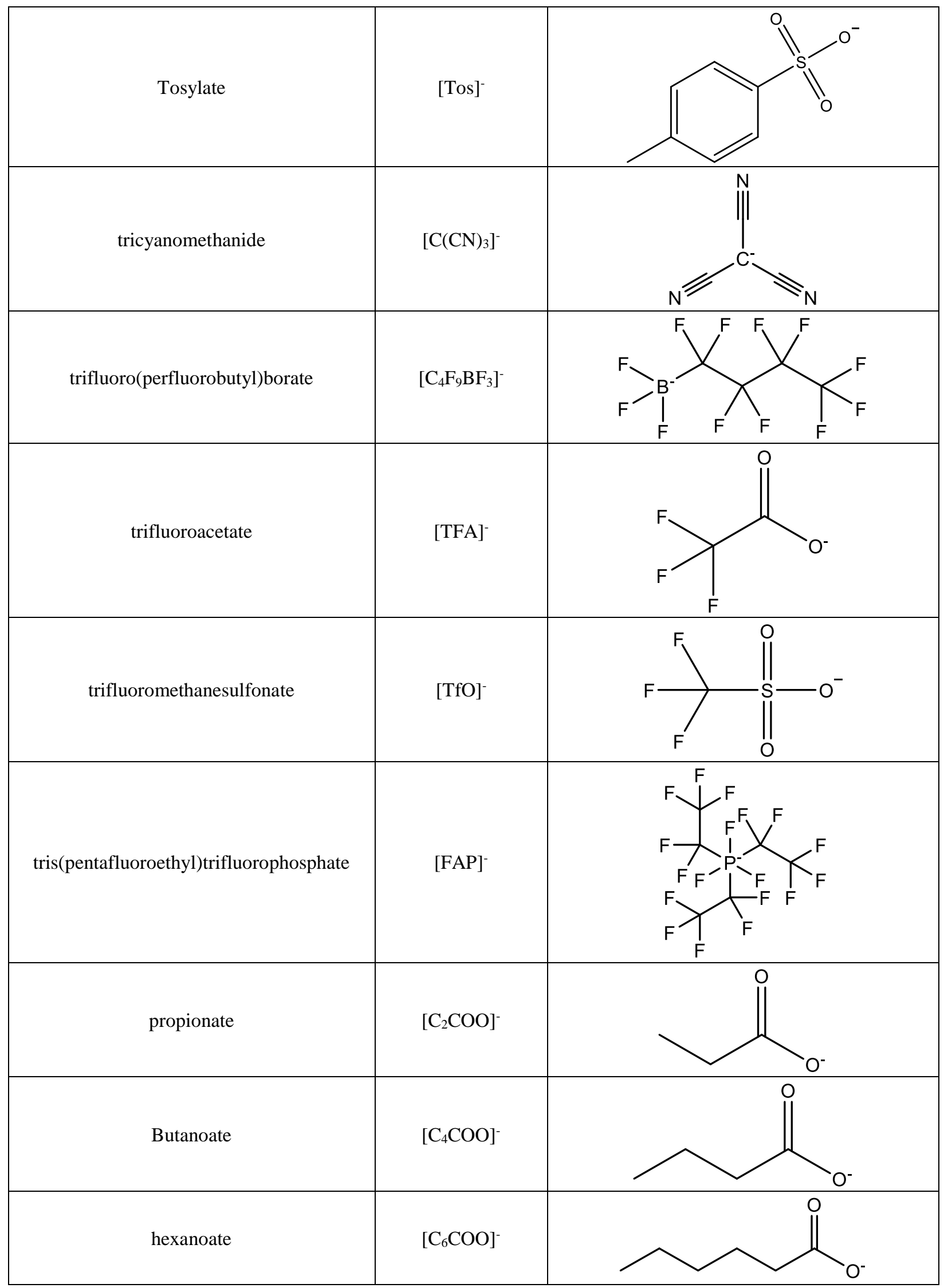




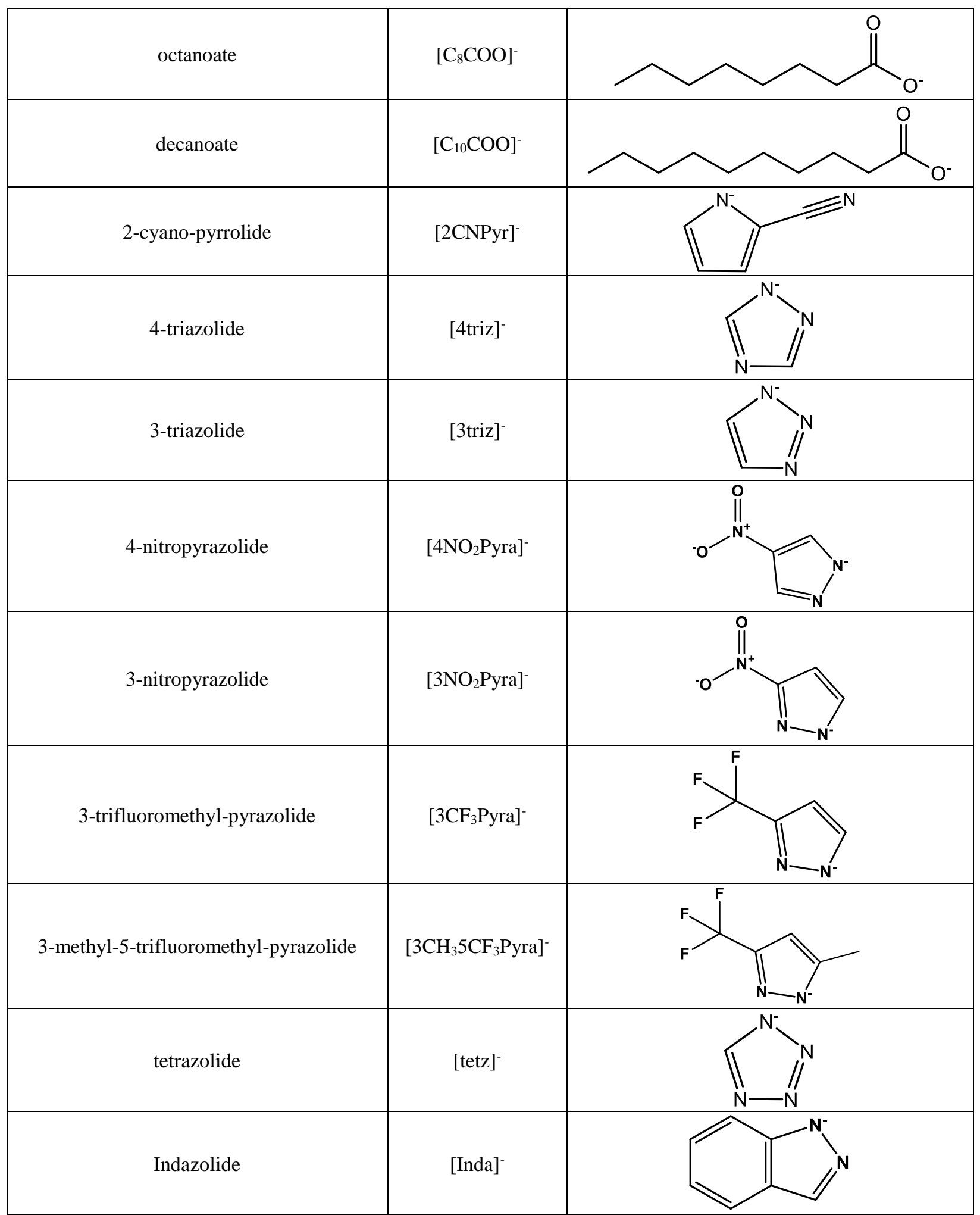


Table S1c. Solvent names, abbreviations, and 2D structures

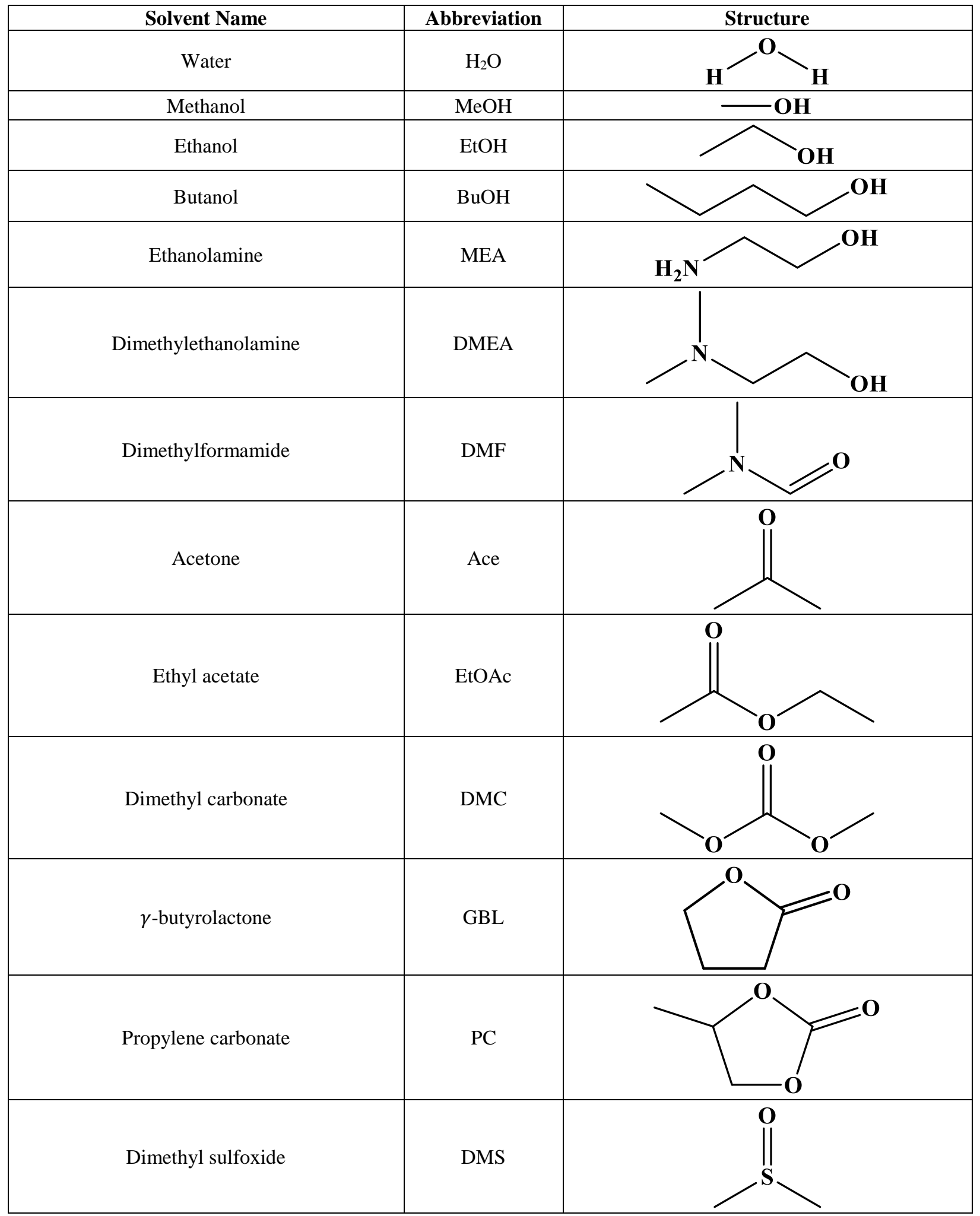




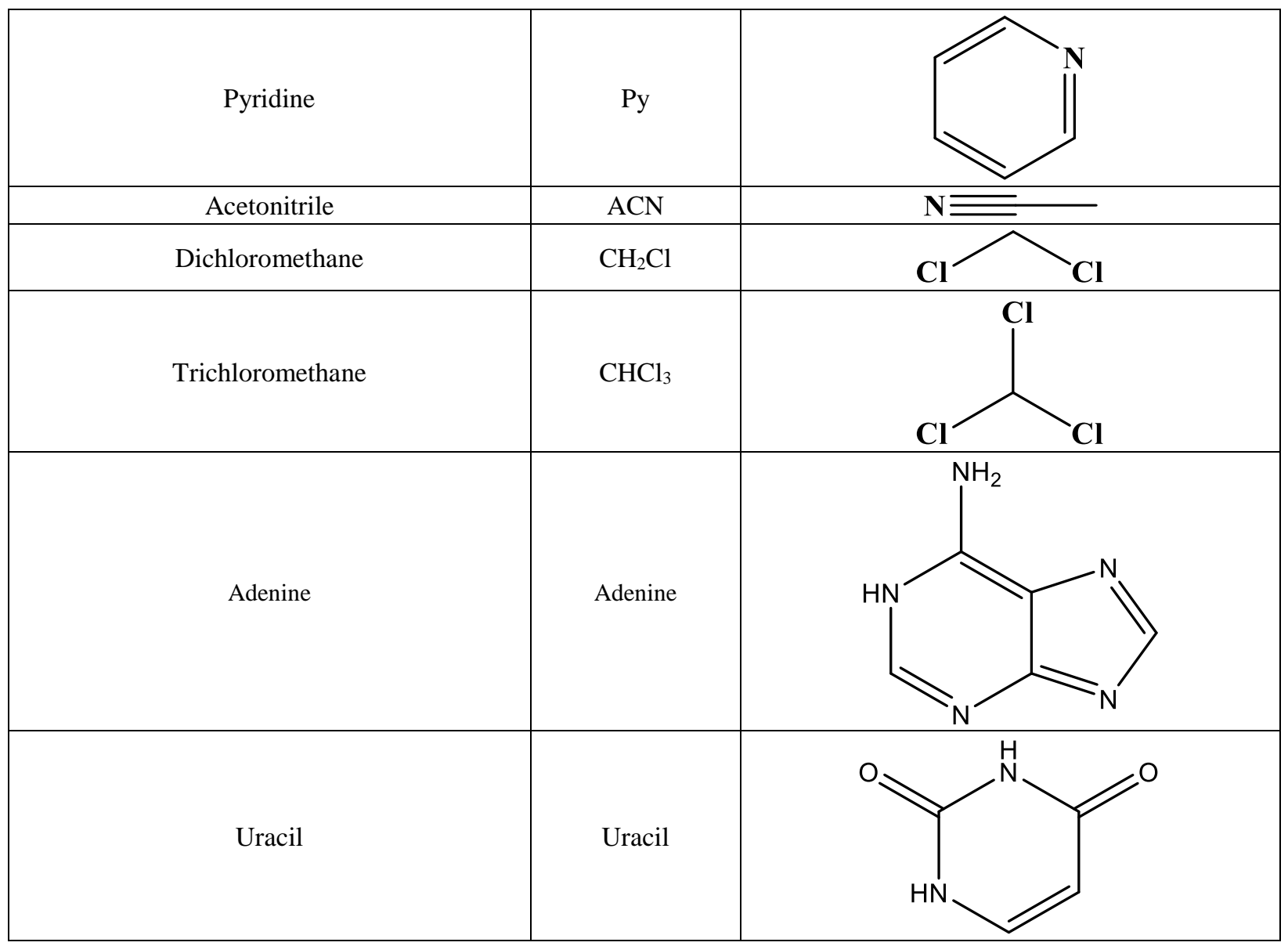


Table S2a. UNIFAC $\boldsymbol{r}$ and $\boldsymbol{q}$ parameters and calculated Stokes radii $\left(\boldsymbol{s}^{+}\right)$for the cations in this work, as well as the UNIFAC group contribution sources used to calculate these values.

\begin{tabular}{|c|c|c|c|c|c|}
\hline \multicolumn{6}{|c|}{ Ammonium Cations } \\
\hline Cation & Abbreviation & $r$ & $q$ & $s^{+}[\mathrm{m}]$ & Ref \\
\hline methylammonium & {$\left[\mathrm{C}_{1} \mathrm{NH}_{3}\right]^{+}$} & 1.84 & 2.23 & $1.51 \mathrm{E}-10$ & 1,2 \\
\hline ethylammonium & {$\left[\mathrm{C}_{2} \mathrm{NH}_{3}\right]^{+}$} & 2.48 & 2.93 & $1.54 \mathrm{E}-10$ & 1,2 \\
\hline propylammonium & {$\left[\mathrm{C}_{3} \mathrm{NH}_{3}\right]^{+}$} & 3.11 & 3.64 & $1.55 \mathrm{E}-10$ & 1,2 \\
\hline butylammonium & {$\left[\mathrm{C}_{4} \mathrm{NH}_{3}\right]^{+}$} & 3.74 & 4.35 & $1.57 \mathrm{E}-10$ & 1,2 \\
\hline pentylammonium & {$\left[\mathrm{C}_{5} \mathrm{NH}_{3}\right]^{+}$} & 4.37 & 5.06 & $1.57 \mathrm{E}-10$ & 1,2 \\
\hline 2-methylpropylammonium & {$\left[\mathrm{iC}_{3} \mathrm{NH}_{3}\right]^{+}$} & 3.74 & 4.35 & $1.57 \mathrm{E}-10$ & 1,2 \\
\hline 2-methylbutylammonium & {$\left[{ }_{2 i} \mathrm{C}_{4} \mathrm{NH}_{3}\right]^{+}$} & 4.37 & 5.06 & $1.57 \mathrm{E}-10$ & 1,2 \\
\hline diethylammonium & {$\left[\mathrm{C}_{2} \mathrm{C}_{2} \mathrm{NH}_{2}\right]^{+}$} & 4.00 & 4.86 & $1.50 \mathrm{E}-10$ & 1,2 \\
\hline ethyldiisopropylammonium & {$\left[\left(\mathrm{iC}_{2}\right)_{3} \mathrm{NH}\right]^{+}$} & 7.67 & 6.63 & $2.10 \mathrm{E}-10$ & 1,2 \\
\hline ethanolammonium & {$\left[2 \mathrm{OHNH}_{3}\right]^{+}$} & 3.56 & 3.44 & $1.88 \mathrm{E}-10$ & 1,2 \\
\hline diethanolammonium & {$\left[(2 \mathrm{OH})_{2} \mathrm{NH}_{2}\right]^{+}$} & 5.17 & 4.68 & $2.01 \mathrm{E}-10$ & 1,2 \\
\hline 2-hydroxypropanaminium & {$\left[1 \mathrm{CHOH} 1 \mathrm{NH}_{3}\right]^{+}$} & 4.23 & 3.98 & $1.94 \mathrm{E}-10$ & 1,2 \\
\hline $\mathrm{N}, \mathrm{N}$-dimethylformamide & {$[\mathrm{DMF}]^{+}$} & 4.40 & 4.04 & $1.98 \mathrm{E}-10$ & 1,2 \\
\hline $\mathrm{N}, \mathrm{N}$-dimethylpropionamidium & {$[\mathrm{DMP}]^{+}$} & 6.42 & 5.66 & $2.06 \mathrm{E}-10$ & 1,2 \\
\hline butyltrimethylammonium & {$\left[\mathrm{N}_{1114}\right]^{+}$} & 5.91 & 5.10 & $2.11 \mathrm{E}-10$ & 3,4 \\
\hline hexyltrimethylammonium & {$\left[\mathrm{N}_{1116}\right]^{+}$} & 7.26 & 6.18 & $2.14 \mathrm{E}-10$ & 3,4 \\
\hline heptyltrimethylammonium & {$\left[\mathrm{N}_{1117}\right]^{+}$} & 7.94 & 6.72 & $2.15 \mathrm{E}-10$ & 3,4 \\
\hline octyltrimethylammonium & {$\left[\mathrm{N}_{1118}\right]^{+}$} & 8.61 & 7.26 & $2.16 \mathrm{E}-10$ & 3,4 \\
\hline trimethyl(dodecyl)ammonium & {$\left[\mathrm{N}_{11112}\right]^{+}$} & 11.31 & 9.42 & $2.18 \mathrm{E}-10$ & 3,4 \\
\hline trimethyl(tetradecyl)ammonium & {$\left[\mathrm{N}_{11114}\right]^{+}$} & 12.66 & 10.50 & $2.19 \mathrm{E}-10$ & 3,4 \\
\hline tetraethylammonium & {$\left[\mathrm{N}_{2222}\right]^{+}$} & 6.59 & 5.64 & $2.12 \mathrm{E}-10$ & 3,4 \\
\hline hexyltriethylammonium & {$\left[\mathrm{N}_{2226}\right]^{+}$} & 9.29 & 7.80 & $2.17 \mathrm{E}-10$ & 3,4 \\
\hline heptyltriethylammonium & {$\left[\mathrm{N}_{2227}\right]^{+}$} & 9.96 & 8.34 & $2.17 \mathrm{E}-10$ & 3,4 \\
\hline octyltriethylammonium & {$\left[\mathrm{N}_{2228}\right]^{+}$} & 10.63 & 8.88 & $2.18 \mathrm{E}-10$ & 3,4 \\
\hline triethyl(decyl)ammonium & {$\left[\mathrm{N}_{22210}\right]^{+}$} & 11.98 & 9.96 & $2.19 \mathrm{E}-10$ & 3,4 \\
\hline triethyl(tetradecyl)ammonium & {$\left[\mathrm{N}_{22214}\right]^{+}$} & 14.68 & 12.12 & $2.20 \mathrm{E}-10$ & 3,4 \\
\hline tetrapropylammonium & {$\left[\mathrm{N}_{3333}\right]^{+}$} & 9.29 & 7.80 & $2.17 \mathrm{E}-10$ & 3,4 \\
\hline ethyltributylphosphonium & {$\left[\mathrm{N}_{4442}\right]^{+}$} & 10.63 & 8.88 & $2.18 \mathrm{E}-10$ & 3,4 \\
\hline tetrabutylammonium & {$\left[\mathrm{N}_{4444}\right]^{+}$} & 11.98 & 9.96 & $2.19 \mathrm{E}-10$ & 3,4 \\
\hline tributylhexylammonium & {$\left[\mathrm{N}_{4446}\right]^{+}$} & 13.33 & 11.04 & $2.20 \mathrm{E}-10$ & 3,4 \\
\hline tributylheptylammonium & {$\left[\mathrm{N}_{4447}\right]^{+}$} & 14.01 & 11.58 & $2.20 \mathrm{E}-10$ & 3,4 \\
\hline tributyloctylammonium & {$\left[\mathrm{N}_{4448}\right]^{+}$} & 14.68 & 12.12 & $2.20 \mathrm{E}-10$ & 3,4 \\
\hline tetrapentylammonium & {$\left[\mathrm{N}_{5555}\right]^{+}$} & 14.68 & 12.12 & $2.20 \mathrm{E}-10$ & 3,4 \\
\hline tetrahexylammonium & {$\left[\mathrm{N}_{6666}\right]^{+}$} & 17.38 & 14.28 & $2.21 \mathrm{E}-10$ & 3,4 \\
\hline $\mathrm{N}, \mathrm{N}, \mathrm{N}$-triheptyl-1-heptanaminium & {$\left[\mathrm{N}_{4448}\right]^{+}$} & 20.08 & 16.44 & $2.22 \mathrm{E}-10$ & 3,4 \\
\hline
\end{tabular}




\begin{tabular}{|c|c|c|c|c|c|}
\hline methyltrioctylammonium & {$\left[\mathrm{N}_{1888}\right]^{+}$} & 18.05 & 14.82 & $2.22 \mathrm{E}-10$ & 3,4 \\
\hline tetraoctylammonium & {$\left[\mathrm{N}_{8888}\right]^{+}$} & 22.77 & 18.60 & $2.23 \mathrm{E}-10$ & 3,4 \\
\hline N-ethyl-N,N-dimethyl-1-propanaminium & {$\left[\mathrm{N}_{1123}\right]^{+}$} & 5.91 & 5.10 & $2.11 \mathrm{E}-10$ & 3,4 \\
\hline $\mathrm{N}, \mathrm{N}$-dimethyl-N-ethyl-N-pentylammonium & {$\left[\mathrm{N}_{1125}\right]^{+}$} & 7.26 & 6.18 & $2.14 \mathrm{E}-10$ & 3,4 \\
\hline N-ethyl-N,N-dimethylheptanaminium & {$\left[\mathrm{N}_{1127}\right]^{+}$} & 8.61 & 7.26 & $2.16 \mathrm{E}-10$ & 3,4 \\
\hline $\mathrm{N}, \mathrm{N}$-diethyl-N-methylpropan-1-aminium & {$\left[\mathrm{N}_{1223}\right]^{+}$} & 6.59 & 5.64 & $2.12 \mathrm{E}-10$ & 3,4 \\
\hline $\mathrm{N}, \mathrm{N}$-diethyl-N-methylbutan-1-aminium & {$\left[\mathrm{N}_{1224}\right]^{+}$} & 7.26 & 6.18 & $2.14 \mathrm{E}-10$ & 3,4 \\
\hline benzyltrimethylammonium & {$\left[\mathrm{N}_{111 \mathrm{Ben}}\right]^{+}$} & 7.22 & 5.70 & $2.31 \mathrm{E}-10$ & 3,4 \\
\hline benzyltripropylammonium & {$\left[\mathrm{N}_{333 \mathrm{Ben}}\right]^{+}$} & 11.26 & 8.94 & $2.29 \mathrm{E}-10$ & 3,4 \\
\hline benzyltributylammonium & {$\left[\mathrm{N}_{444 \mathrm{Ben}}\right]^{+}$} & 13.29 & 10.56 & $2.29 \mathrm{E}-10$ & 3,4 \\
\hline 2-hydroxy-N,N,N-trimethylethanaminium & {$\left[\mathrm{N}_{111(2 \mathrm{OH})}\right]^{+}$} & 5.34 & 4.92 & $1.98 \mathrm{E}-10$ & 3,4 \\
\hline 2-methoxy-N,N,N-trimethylethanaminium & {$\left[\mathrm{N}_{111(2 \mathrm{O})}\right]^{+}$} & 5.48 & 4.80 & $2.08 \mathrm{E}-10$ & 3,4 \\
\hline $\mathrm{N}, \mathrm{N}, \mathrm{N}$-triethyl-2-methoxyethanaminium & {$\left[\mathrm{N}_{222(2 \mathrm{O})}\right]^{+}$} & 7.51 & 6.42 & $2.13 \mathrm{E}-10$ & 3,4 \\
\hline $\begin{array}{l}\text { N-ethyl-N,N-dimethyl-2- } \\
\text { methoxyethylammonium }\end{array}$ & {$\left[\mathrm{N}_{112(2 \mathrm{O})}\right]^{+}$} & 6.16 & 5.34 & $2.10 \mathrm{E}-10$ & 3,4 \\
\hline $\begin{array}{l}\mathrm{N}, \mathrm{N} \text {-diethyl-2-methoxy-N- } \\
\text { methylethanaminium }\end{array}$ & {$\left[\mathrm{N}_{122(2 \mathrm{O})}\right]^{+}$} & 6.83 & 5.88 & $2.11 \mathrm{E}-10$ & 3,4 \\
\hline $\begin{array}{l}\text { N-ethyl-2-(2-methoxyethoxy)-N,N- } \\
\text { dimethylethanaminium }\end{array}$ & {$\left[\mathrm{N}_{122(2 \mathrm{O} 2 \mathrm{O})]^{+}}\right.$} & 7.75 & 6.66 & $2.12 \mathrm{E}-10$ & 3,4 \\
\hline $\begin{array}{c}\text { 2-(acetyloxy)-N-ethyl-N,N- } \\
\text { dimethylethanaminium }\end{array}$ & {$\left[\mathrm{N}_{122(2 \mathrm{OCO})]^{+}}\right.$} & 6.92 & 5.98 & $2.10 \mathrm{E}-10$ & 3,4 \\
\hline \multicolumn{6}{|c|}{ Phosphonium Cations } \\
\hline Cation & Abbreviation & $r$ & $q$ & $s^{+}[\mathrm{m}]$ & Ref \\
\hline triethylbutylphosphonium & {$\left[\mathrm{P}_{2224}\right]^{+}$} & 8.24 & 6.70 & $2.24 \mathrm{E}-10$ & 4 \\
\hline triethylpentylphosphonium & {$\left[\mathrm{P}_{2225}\right]^{+}$} & 8.92 & 7.24 & $2.24 \mathrm{E}-10$ & 4 \\
\hline triethyloctylphosphonium & {$\left[\mathrm{P}_{2228}\right]^{+}$} & 10.94 & 8.86 & $2.25 \mathrm{E}-10$ & 4 \\
\hline tributylmethylphosphonium & {$\left[\mathrm{P}_{4441}\right]^{+}$} & 10.27 & 8.32 & $2.25 \mathrm{E}-10$ & 4 \\
\hline tributylethylphosphonium & {$\left[\mathrm{P}_{4442}\right]^{+}$} & 10.94 & 8.86 & $2.25 \mathrm{E}-10$ & 4 \\
\hline tetrabutylphosphonium & {$\left[\mathrm{P}_{4444}\right]^{+}$} & 12.29 & 9.94 & $2.25 \mathrm{E}-10$ & 4 \\
\hline tributylhexylphosphonium & {$\left[\mathrm{P}_{4446}\right]^{+}$} & 13.64 & 11.02 & $2.25 \mathrm{E}-10$ & 4 \\
\hline tributyloctylphosphonium & {$\left[\mathrm{P}_{4448}\right]^{+}$} & 14.99 & 12.10 & $2.25 \mathrm{E}-10$ & 4 \\
\hline tributyl(decyl)phosphonium & {$\left[\mathrm{P}_{44410}\right]^{+}$} & 16.34 & 13.18 & $2.26 \mathrm{E}-10$ & 4 \\
\hline trihexyl(tetradecyl)phosphonium & {$\left[\mathrm{P}_{66614}\right]^{+}$} & 23.08 & 18.58 & $2.26 \mathrm{E}-10$ & 4 \\
\hline triethyl(2-methoxyethyl)phosphonium & {$\left[\mathrm{P}_{222(2 \mathrm{O})}\right]^{+}$} & 7.81 & 6.40 & $2.22 \mathrm{E}-10$ & 4 \\
\hline (2-ethoxyethyl)triethylphosphonium & {$\left[\mathrm{P}_{222(2 \mathrm{O} 2)}\right]^{+}$} & 8.49 & 6.94 & $2.22 \mathrm{E}-10$ & 4 \\
\hline (3-aminopropyl)tributylphosphonium & {$\left[\mathrm{P}_{444(3 \mathrm{NH} 2)}\right]^{+}$} & 12.37 & 9.89 & $2.28 \mathrm{E}-10$ & 1,4 \\
\hline \multicolumn{6}{|c|}{ Morpholinium Cations } \\
\hline Cation & Abbreviation & $r$ & $q$ & $s^{+}[\mathrm{m}]$ & Ref \\
\hline 4-ethyl-4-methylmorpholinium & {$\left[\mathrm{C}_{2} \mathrm{mMor}\right]^{+}$} & 9.10 & 7.61 & $2.18 \mathrm{E}-10$ & 4,5 \\
\hline 4-butyl-4-methylmorpholinium & {$\left[\mathrm{C}_{4} \mathrm{mMor}\right]^{+}$} & 5.06 & 4.37 & $2.11 \mathrm{E}-10$ & 4,5 \\
\hline 4-hexyl-4-methylmorpholinium & {$\left[\mathrm{C}_{6} \mathrm{mMor}\right]^{+}$} & 6.41 & 5.45 & $2.14 \mathrm{E}-10$ & 4,5 \\
\hline
\end{tabular}




\begin{tabular}{|c|c|c|c|c|c|}
\hline $\begin{array}{l}\text { 4-methyl-4-octylmorpholinium } \\
\text { 4-(2-methoxyethyl)-4-methylmorpholinium }\end{array}$ & $\begin{array}{c}{\left[\mathrm{C}_{8} \mathrm{mMor}\right]_{+}} \\
{[(2 \mathrm{O} 1) \mathrm{mMor}]^{+}}\end{array}$ & $\begin{array}{l}7.75 \\
5.98\end{array}$ & $\begin{array}{l}6.53 \\
5.15\end{array}$ & $\begin{array}{l}2.16 \mathrm{E}-10 \\
2.11 \mathrm{E}-10\end{array}$ & $\begin{array}{l}4,5 \\
4,5\end{array}$ \\
\hline \multicolumn{6}{|c|}{ Pyrrolidinium Cations } \\
\hline Cation & Abbreviation & $r$ & $q$ & $s^{+}[\mathbf{m}]$ & Ref \\
\hline pyrrolidinium & [pyrr] $^{+}$ & 2.79 & 2.77 & $1.83 \mathrm{E}-10$ & 1 \\
\hline 1-ethyl-1-methylpyrrolidinium & {$\left[\mathrm{C}_{2} \mathrm{mpyrr}\right]^{+}$} & 4.68 & 5.60 & $1.52 \mathrm{E}-10$ & 1 \\
\hline 1-propyl-1-methylpyrrolidinium & {$\left[\mathrm{C}_{3} \mathrm{mpyrr}\right]^{+}$} & 5.32 & 6.31 & $1.53 \mathrm{E}-10$ & 1 \\
\hline 1-butyl-1-methylpyrrolidinium & {$\left[\mathrm{C}_{4} \mathrm{mpyrr}\right]^{+}$} & 5.95 & 7.02 & $1.54 \mathrm{E}-10$ & 1 \\
\hline 1-hexyl-1-methylpyrrolidinium & {$\left[\mathrm{C}_{6} \mathrm{mpyrr}\right]^{+}$} & 7.21 & 8.43 & $1.56 \mathrm{E}-10$ & 1 \\
\hline N-methyl-N-octylpyrrolidinium & {$\left[\mathrm{C}_{8} \mathrm{mpyrr}\right]^{+}$} & 8.48 & 9.85 & $1.57 \mathrm{E}-10$ & 1 \\
\hline 1-(2-methoxyethyl)-1-methylpyrrolidinium & {$[2 \mathrm{O} 1 \mathrm{mpyrr}]^{+}$} & 5.83 & 6.85 & $1.55 \mathrm{E}-10$ & 1 \\
\hline 1-(methoxymethyl)-1-methylpyrrolidinium & {$[1 \mathrm{O} 1 \mathrm{mpyrr}]^{+}$} & 5.20 & 6.14 & $1.54 \mathrm{E}-10$ & 1 \\
\hline
\end{tabular}

Pyridinium Cations

\begin{tabular}{cccccc}
\hline Cation & Abbreviation & $\boldsymbol{r}$ & $\boldsymbol{q}$ & $\boldsymbol{s}^{+}[\mathbf{m}]$ & Ref \\
\hline 1-methylpyridinium & {$\left[\mathrm{mPy}^{+}\right.$} & 2.74 & 3.57 & $1.40 \mathrm{E}-10$ & 1 \\
1-ethylpyridinium & {$\left[\mathrm{C}_{2} \mathrm{Py}\right]^{+}$} & 3.37 & 4.28 & $1.44 \mathrm{E}-10$ & 1 \\
1-propylpyridinium & {$\left[\mathrm{C}_{3} \mathrm{Py}^{+}\right.$} & 4.01 & 4.99 & $1.46 \mathrm{E}-10$ & 1 \\
1-butylpyridinium & {$\left[\mathrm{C}_{4} \mathrm{Py}\right]^{+}$} & 4.64 & 5.70 & $1.48 \mathrm{E}-10$ & 1 \\
1-pentylpyridinium & {$\left[\mathrm{C}_{5} \mathrm{Py}\right]^{+}$} & 5.27 & 6.40 & $1.50 \mathrm{E}-10$ & 1 \\
1-hexylpyridinium & {$\left[\mathrm{C}_{6} \mathrm{Py}^{+}\right.$} & 5.90 & 7.11 & $1.51 \mathrm{E}-10$ & 1 \\
1-ethyl-2-methylpyridinium & {$\left[\mathrm{C}_{2} \mathrm{mPy}_{12}\right]^{+}$} & 4.38 & 5.28 & $1.51 \mathrm{E}-10$ & 1 \\
1-ethyl-3-methylpyridinium & {$\left[\mathrm{C}_{2} \mathrm{mPy}_{13}\right]^{+}$} & 4.38 & 5.28 & $1.51 \mathrm{E}-10$ & 1 \\
1-ethyl-4-methylpyridinium & {$\left[\mathrm{C}_{2} \mathrm{mPy}_{14}\right]^{+}$} & 4.38 & 5.28 & $1.51 \mathrm{E}-10$ & 1 \\
1-propyl-2-methylpyridinium & {$\left[\mathrm{C}_{3} \mathrm{mPy}_{12}\right]^{+}$} & 5.02 & 5.98 & $1.53 \mathrm{E}-10$ & 1 \\
1-propyl-3-methylpyridinium & {$\left[\mathrm{C}_{3} \mathrm{mPy}_{13}\right]^{+}$} & 5.02 & 5.98 & $1.53 \mathrm{E}-10$ & 1 \\
1-butyl-2-methylpyridinium & {$\left[\mathrm{C}_{4} \mathrm{mPy}_{12}\right]^{+}$} & 5.65 & 6.69 & $1.54 \mathrm{E}-10$ & 1 \\
1-butyl-3-methylpyridinium & {$\left[\mathrm{C}_{3} \mathrm{mPy}_{13}\right]^{+}$} & 5.65 & 6.69 & $1.54 \mathrm{E}-10$ & 1 \\
1-butyl-4-methylpyridinium & {$\left[\mathrm{C}_{3} \mathrm{mPy}_{14}\right]^{+}$} & 5.65 & 6.69 & $1.54 \mathrm{E}-10$ & 1 \\
1-hexyl-3-methylpyridinium & {$\left[\mathrm{C}_{6} \mathrm{mPy}_{13}\right]^{+}$} & 6.91 & 8.11 & $1.55 \mathrm{E}-10$ & 1 \\
1-hexyl-4-methylpyridinium & {$\left[\mathrm{C}_{6} \mathrm{mPy}_{14}\right]^{+}$} & 6.91 & 8.11 & $1.55 \mathrm{E}-10$ & 1 \\
N-octyl-3-methylpyridinium & {$\left[\mathrm{C}_{8} \mathrm{mPy}_{13}\right]^{+}$} & 8.18 & 9.52 & $1.56 \mathrm{E}-10$ & 1 \\
1-(2-hydroxyethyl)-pyridinium & {$\left[2 \mathrm{OHPy}_{1}\right]^{+}$} & 4.60 & 4.82 & $1.74 \mathrm{E}-10$ & 1 \\
N-butyronitrilepyridinium & {$\left[2 \mathrm{CNPy}_{1}\right]^{+}$} & 4.93 & 5.45 & $1.65 \mathrm{E}-10$ & 1 \\
\hline
\end{tabular}

\begin{tabular}{cccccc}
\hline \multicolumn{7}{c}{ Imidazolium Cations } & & & & \\
\hline Cation & Abbreviation & $\boldsymbol{r}$ & $\boldsymbol{q}$ & $\boldsymbol{s}^{+}[\mathbf{m}]$ & Ref \\
\hline 1-methylimidazolium & {$[\mathrm{mim}]^{+}$} & 2.48 & 2.76 & $1.63 \mathrm{E}-10$ & 1 \\
1,3-dimethylimidazolium & {$[\mathrm{mmim}]^{+}$} & 3.11 & 3.82 & $1.48 \mathrm{E}-10$ & 1 \\
1-ethyl-3-methylimidazolium & {$\left[\mathrm{C}_{2} \mathrm{mim}\right]^{+}$} & 3.74 & 4.53 & $1.50 \mathrm{E}-10$ & 1 \\
1-propyl-3-methylimidazolium & {$\left[\mathrm{C}_{3} \mathrm{mim}\right]^{+}$} & 4.37 & 5.24 & $1.52 \mathrm{E}-10$ & 1 \\
1-butyl-3-methylimidazolium & {$\left[\mathrm{C}_{4} \mathrm{mim}\right]^{+}$} & 5.01 & 5.95 & $1.53 \mathrm{E}-10$ & 1
\end{tabular}




\begin{tabular}{|c|c|c|c|c|c|}
\hline 1-pentyl-3-methylimidazolium & {$\left[\mathrm{C}_{5} \mathrm{mim}\right]^{+}$} & 5.64 & 6.65 & $1.54 \mathrm{E}-10$ & 1 \\
\hline 1-hexyl-3-methylimidazolium & {$\left[\mathrm{C}_{6} \mathrm{mim}\right]^{+}$} & 6.27 & 7.36 & $1.55 \mathrm{E}-10$ & 1 \\
\hline 1-octyl-3-methylimidazolium & {$\left[\mathrm{C}_{8} \mathrm{mim}\right]^{+}$} & 7.54 & 8.78 & $1.56 \mathrm{E}-10$ & 1 \\
\hline 1-decyl-3-methylimidazolium & {$\left[\mathrm{C}_{10} \mathrm{mim}\right]^{+}$} & 8.80 & 10.19 & $1.57 \mathrm{E}-10$ & 1 \\
\hline 1-dodecyl-3-methylimidazolium & {$\left[\mathrm{C}_{12} \mathrm{mim}\right]^{+}$} & 10.07 & 11.61 & $1.58 \mathrm{E}-10$ & 1 \\
\hline 1-hexadecyl-3-methylimidazolium & {$\left[\mathrm{C}_{16} \mathrm{mim}\right]^{+}$} & 13.86 & 15.86 & $1.59 \mathrm{E}-10$ & 1 \\
\hline 1-butyl-3-ethylimidazolium & {$\left[\mathrm{C}_{4} \mathrm{C}_{2} \mathrm{im}\right]^{+}$} & 5.64 & 6.65 & $1.54 \mathrm{E}-10$ & 1 \\
\hline 1-pentyl-3-butylimidazolium & {$\left[\mathrm{C}_{5} \mathrm{C}_{4} \mathrm{im}\right]^{+}$} & 7.54 & 8.78 & $1.56 \mathrm{E}-10$ & 1 \\
\hline 1-isopenty-3-butylimidazolium & {$\left[\mathrm{iC}_{5} \mathrm{C}_{4} \mathrm{im}\right]^{+}$} & 8.17 & 9.84 & $1.51 \mathrm{E}-10$ & 1 \\
\hline 1-ethyl-2,3-dimethylimidazolium & {$\left[\mathrm{C}_{2} \mathrm{mmim}\right]^{+}$} & 3.90 & 4.57 & $1.55 \mathrm{E}-10$ & 1 \\
\hline 1-propyl-2,3-dimethylimidazolium & {$\left[\mathrm{C}_{3} \mathrm{mmim}\right]^{+}$} & 4.53 & 5.28 & $1.56 \mathrm{E}-10$ & 1 \\
\hline 1-butyl-2,3-dimethylimidazolium & {$\left[\mathrm{C}_{4} \mathrm{mmim}\right]^{+}$} & 5.16 & 5.99 & $1.57 \mathrm{E}-10$ & 1 \\
\hline 1-pentyl-2,3-dimethylimidazolium & {$\left[\mathrm{C}_{5} \mathrm{mmim}\right]^{+}$} & 5.79 & 6.69 & $1.58 \mathrm{E}-10$ & 1 \\
\hline 1-hexyl-2,3-dimethylimidazolium & {$\left[\mathrm{C}_{6} \mathrm{mmim}\right]^{+}$} & 6.43 & 7.40 & $1.58 \mathrm{E}-10$ & 1 \\
\hline 1-allyl-3-methylimidazolium & {$[\text { Amim }]^{+}$} & 3.74 & 3.86 & $1.76 \mathrm{E}-10$ & 1 \\
\hline 1-cyclopentyl-3-butylimidazolium & {$\left[\mathrm{cC}_{5} \mathrm{C}_{4} \mathrm{im}\right]^{+}$} & 7.58 & 8.45 & $1.63 \mathrm{E}-10$ & 1 \\
\hline 1-(1-cyanomethyl)-3-methylimidazolium & {$[1 \mathrm{CNmim}]^{+}$} & 4.03 & 3.93 & $1.87 \mathrm{E}-10$ & 1 \\
\hline 1-butyronitrile-3-methylimidazolium & {$[3 \mathrm{CNmim}]^{+}$} & 5.30 & 5.34 & $1.80 \mathrm{E}-10$ & 1 \\
\hline $\begin{array}{l}\text { 3-(2-(butylamino)-2-oxoethyl)-1- } \\
\text { ethylimidazolium }\end{array}$ & {$\left[(\mathrm{HCONH}) 4 \mathrm{C}_{2} \mathrm{im}\right]^{+}$} & 7.14 & 7.73 & $1.68 \mathrm{E}-10$ & 1 \\
\hline 1-(2-hydroxyethyl)-3-methylimidazolium & {$[2 \mathrm{OHmim}]^{+}$} & 4.97 & 5.07 & $1.78 \mathrm{E}-10$ & 1 \\
\hline \multicolumn{6}{|c|}{ Piperidinium Cations } \\
\hline Cation & Abbreviation & $r$ & $q$ & $s^{+}[\mathrm{m}]$ & Ref \\
\hline 1-ethyl-1-methylpiperidinium & {$\left[\mathrm{C}_{2} \mathrm{mpip}\right]^{+}$} & 5.42 & 4.79 & $2.06 \mathrm{E}-10$ & 4,5 \\
\hline 1-propyl-1-methylpiperidinium & {$\left[\mathrm{C}_{3} \mathrm{mpip}\right]^{+}$} & 6.09 & 5.33 & $2.08 \mathrm{E}-10$ & 4,5 \\
\hline 1-butyl-1-methylpiperidinium & {$\left[\mathrm{C}_{4} \mathrm{mpip}\right]^{+}$} & 6.77 & 5.87 & $2.10 \mathrm{E}-10$ & 4,5 \\
\hline 1-hexyl-1-methylpiperidinium & {$\left[\mathrm{C}_{6} \text { mpip }\right]^{+}$} & 8.12 & 6.95 & $2.13 \mathrm{E}-10$ & 4,5 \\
\hline 1-octyl-1-metylpiperidinium & {$\left[\mathrm{C}_{8} \mathrm{mpip}\right]^{+}$} & 9.47 & 8.03 & $2.15 \mathrm{E}-10$ & 4,5 \\
\hline 1-(2-methoxyethyl)-1-methylpiperidinium & {$[2 \mathrm{O} 1 \mathrm{mpip}]^{+}$} & 5.98 & 6.63 & $1.64 \mathrm{E}-10$ & 4,5 \\
\hline \multicolumn{6}{|c|}{ Sulfonium Cations } \\
\hline Cation & Abbreviation & $r$ & $q$ & $s^{+}[\mathbf{m}]$ & Ref \\
\hline triethylsulfonium & {$\left[\mathrm{S}_{222}\right]^{+}$} & 4.55 & 5.66 & $1.46 \mathrm{E}-10$ & 1 \\
\hline diethylmethylsulfonium & {$\left[\mathrm{S}_{122}\right]^{+}$} & 4.14 & 4.91 & $1.54 \mathrm{E}-10$ & 1 \\
\hline
\end{tabular}


Table S2b. UNIFAC $\boldsymbol{r}$ and $\boldsymbol{q}$ parameters and calculated Stokes radii $\left(\boldsymbol{s}^{-}\right)$for the anions in this work as well as the UNIFAC group contribution sources used to calculate these values.

\begin{tabular}{|c|c|c|c|c|c|}
\hline Anion & Abbreviation & $r$ & $q$ & $s^{-}[\mathrm{m}]$ & Ref \\
\hline beta.-alaninate & [beta-al] $]^{-}$ & 3.89 & 3.30 & $2.15 \mathrm{E}-10$ & 1 \\
\hline acetate & {$[\mathrm{Ac}]^{-}$} & 2.87 & 2.18 & $2.40 \mathrm{E}-10$ & 4 \\
\hline glycinate & {$\left[\mathrm{Gly}^{-}\right.$} & 3.26 & 2.24 & $2.65 \mathrm{E}-10$ & 1 \\
\hline bis(fluorosulfonyl)imide & {$[\mathrm{FSI}]^{-}$} & 4.49 & 3.83 & $2.13 \mathrm{E}-10$ & 1,3 \\
\hline bis((trifluoromethyl)sulfonyl)imide & {$\left[\mathrm{Tf}_{2} \mathrm{~N}\right]^{-}$} & 5.77 & 4.93 & $2.13 \mathrm{E}-10$ & 3 \\
\hline bis(perfluoroethylsulfonyl)imide & 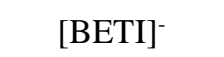 & 7.06 & 6.03 & $2.13 \mathrm{E}-10$ & 1,3 \\
\hline tris(pentafluoroethyl)trifluorophosphate & {$[\mathrm{FAP}]^{-}$} & 9.41 & 7.73 & $2.22 \mathrm{E}-10$ & 4 \\
\hline bromide & {$[\mathrm{Br}]^{-}$} & 0.95 & 0.83 & $2.08 \mathrm{E}-10$ & 3 \\
\hline chloride & {$[\mathrm{Cl}]^{-}$} & 0.77 & 0.72 & $1.94 \mathrm{E}-10$ & 3 \\
\hline iodide & {$[\mathrm{I}]^{-}$} & 1.08 & 0.92 & $2.14 \mathrm{E}-10$ & 1 \\
\hline dicyanamide & {$\left[\mathrm{N}(\mathrm{CN})_{2}\right]^{-}$} & 2.42 & 2.13 & $2.06 \mathrm{E}-10$ & 5 \\
\hline tricyanomethanide & {$\left[\mathrm{C}(\mathrm{CN})_{3}\right]^{-}$} & 3.33 & 2.87 & $2.12 \mathrm{E}-10$ & 5 \\
\hline ethylphosphonate & {$\left[\mathrm{C}_{2} \mathrm{PO}_{3}\right]-$} & 3.39 & 2.91 & $2.12 \mathrm{E}-10$ & 5 \\
\hline butylphosphonate & {$\left[\mathrm{C}_{4} \mathrm{PO}_{3}\right]-$} & 4.74 & 3.99 & $2.16 \mathrm{E}-10$ & 5 \\
\hline hexylphosphonate & {$\left[\mathrm{C}_{6} \mathrm{PO}_{3}\right]-$} & 6.08 & 5.07 & $2.18 \mathrm{E}-10$ & 5 \\
\hline octylphosphonate & {$\left[\mathrm{C}_{8} \mathrm{PO}_{3}\right]-$} & 7.43 & 6.15 & $2.20 \mathrm{E}-10$ & 5 \\
\hline dihydrogenphosphate & {$\left[\mathrm{H}_{2} \mathrm{PO}_{4}\right]^{-}$} & 2.51 & 2.43 & $1.88 \mathrm{E}-10$ & 3 \\
\hline dimethylphosphate & {$\left[\mathrm{C}_{1} \mathrm{C}_{1} \mathrm{PO}_{4}\right]^{-}$} & 3.41 & 3.28 & $1.89 \mathrm{E}-10$ & 3 \\
\hline diethylphosphate & {$\left[\mathrm{C}_{2} \mathrm{C}_{2} \mathrm{PO}_{4}\right]^{-}$} & 6.93 & 4.42 & $2.85 \mathrm{E}-10$ & 6 \\
\hline dibutylphosphate & {$\left[\mathrm{C}_{4} \mathrm{C}_{4} \mathrm{PO}_{4}\right]^{-}$} & 10.41 & 6.32 & $3.00 \mathrm{E}-10$ & 6 \\
\hline methanesulfonate & $\begin{array}{c}{\left[\mathrm{C}_{1} \mathrm{SO}_{3}\right]^{-}} \\
{\left[\mathrm{CF}_{3} \mathrm{SO}_{3}\right]^{-}}\end{array}$ & 2.71 & 2.37 & $2.08 \mathrm{E}-10$ & 5 \\
\hline trifluoromethanesulfonate & $/[\mathrm{TFO}]^{-}$ & 3.47 & 2.98 & $2.12 \mathrm{E}-10$ & 5 \\
\hline hydrogen sulfate & {$\left[\mathrm{HSO}_{4}\right]^{-}$} & 3.23 & 2.37 & $2.48 \mathrm{E}-10$ & 4 \\
\hline methylsulfate & {$\left[\mathrm{C}_{1} \mathrm{SO}_{4}\right]^{-}$} & 3.48 & 3.73 & $1.70 \mathrm{E}-10$ & 5 \\
\hline ethylsulfate & {$\left[\mathrm{C}_{2} \mathrm{SO}_{4}\right]^{-}$} & 4.16 & 4.18 & $1.81 \mathrm{E}-10$ & 3 \\
\hline octylsulfate & {$\left[\mathrm{C}_{8} \mathrm{SO}_{4}\right]^{-}$} & 10.22 & 6.19 & $3.00 \mathrm{E}-10$ & 6 \\
\hline nitrate & {$\left[\mathrm{NO}_{3}\right]^{-}$} & 1.66 & 1.53 & $1.98 \mathrm{E}-10$ & 5 \\
\hline tosylate & 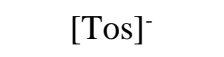 & 5.49 & 4.59 & $2.18 \mathrm{E}-10$ & 5 \\
\hline salicylate & {$\left[\mathrm{Sal}^{-}\right.$} & 4.56 & 4.04 & $2.06 \mathrm{E}-10$ & 1 \\
\hline formate & {$[\mathrm{COO}]^{-}$} & 1.90 & 1.80 & $1.92 \mathrm{E}-10$ & 1 \\
\hline L-alaninate & [L-ala $^{-}$ & 3.89 & 2.95 & $2.41 \mathrm{E}-10$ & 1 \\
\hline lactate & {$[\mathrm{Lac}]^{-}$} & 4.08 & 2.81 & $2.64 \mathrm{E}-10$ & 4 \\
\hline L-leucinate & {$[\mathrm{L}-\mathrm{leu}]^{-}$} & 3.89 & 2.95 & $2.41 \mathrm{E}-10$ & 1 \\
\hline L-prolinate & {$[\mathrm{L}-\text { pro }]^{-}$} & 6.82 & 4.99 & $2.49 \mathrm{E}-10$ & 1 \\
\hline L-serinate & {$[\mathrm{L}-\mathrm{ser}]^{-}$} & 5.12 & 3.49 & $2.68 \mathrm{E}-10$ & 1 \\
\hline thiocyanate & {$[\mathrm{SCN}]^{-}$} & 1.94 & 1.18 & $3.01 \mathrm{E}-10$ & 3 \\
\hline taurate & {$[\mathrm{Tau}]^{-}$} & 4.37 & 3.36 & $2.37 \mathrm{E}-10$ & 1,5 \\
\hline tetracyanoborate & {$\left[\mathrm{B}(\mathrm{CN})_{4}\right]^{-}$} & 4.33 & 3.67 & $2.15 \mathrm{E}-10$ & 5 \\
\hline
\end{tabular}




\begin{tabular}{cccccc} 
tetrafluoroborate & {$\left[\mathrm{BF}_{4}\right]^{-}$} & 1.79 & 1.49 & $2.18 \mathrm{E}-10$ & 3 \\
trifluoro(perfluorobutyl)borate & {$\left[\mathrm{C}_{4} \mathrm{~F}_{9} \mathrm{BF}_{3}\right]^{-}$} & 9.10 & 5.18 & $3.20 \mathrm{E}-10$ & 1,3 \\
trifluoroacetate & {$\left[\mathrm{TFA}^{-}\right.$} & 3.18 & 3.22 & $1.80 \mathrm{E}-10$ & 3 \\
hexafluorophosphate & {$\left[\mathrm{PF}_{6}\right]^{-}$} & 7.06 & 6.58 & $1.95 \mathrm{E}-10$ & 3 \\
hydrogen carbonate & {$\left[\mathrm{HCO}_{3}\right]^{-}$} & 8.08 & 8.68 & $1.69 \mathrm{E}-10$ & 7 \\
propionate & {$\left[\mathrm{C}_{2} \mathrm{COO}\right]^{-}$} & 2.87 & 2.67 & $1.95 \mathrm{E}-10$ & 1 \\
butanoate & {$\left[\mathrm{C}_{4} \mathrm{COO}\right]^{-}$} & 3.50 & 3.38 & $1.89 \mathrm{E}-10$ & 1 \\
hexanoate & {$\left[\mathrm{C}_{6} \mathrm{COO}\right]^{-}$} & 4.76 & 4.79 & $1.81 \mathrm{E}-10$ & 1 \\
octanoate & {$\left[\mathrm{C}_{8} \mathrm{COO}\right]^{-}$} & 6.03 & 6.21 & $1.77 \mathrm{E}-10$ & 1 \\
decanoate & {$\left[\mathrm{C}_{10} \mathrm{COO}^{-}\right.$} & 7.29 & 7.63 & $1.74 \mathrm{E}-10$ & 1 \\
perfluoropentanoate & {$\left[\mathrm{C}_{4} \mathrm{~F}_{9} \mathrm{COO}^{-}\right.$} & 6.74 & 5.46 & $2.25 \mathrm{E}-10$ & 1 \\
trifluoro(perfluorobutyl)borate & {$\left[\mathrm{C}_{4} \mathrm{~F}_{9} \mathrm{BF}_{3}\right]^{-}$} & 6.92 & 6.05 & $2.08 \mathrm{E}-10$ & 1,3 \\
trifluoro(perfluoroethyl)borate & {$\left[\mathrm{C}_{2} \mathrm{~F}_{5} \mathrm{BF}_{3}\right]^{-}$} & 4.35 & 3.86 & $2.05 \mathrm{E}-10$ & 1,3 \\
trifluoro(perfluoropropyl)borate & {$\left[\mathrm{C}_{3} \mathrm{~F}_{7} \mathrm{BF}_{3}\right]^{-}$} & 5.64 & 4.96 & $2.07 \mathrm{E}-10$ & 1,3 \\
trifluoro(trifluoromethyl)borate & {$\left[\mathrm{CF}_{3} \mathrm{BF}_{3}\right]^{-}$} & 3.07 & 2.76 & $2.02 \mathrm{E}-10$ & 1,3 \\
\hline
\end{tabular}


Data Processing and Ion Dissociation Calculation Methods:

Neat IL Ion Dissociation Calculations:

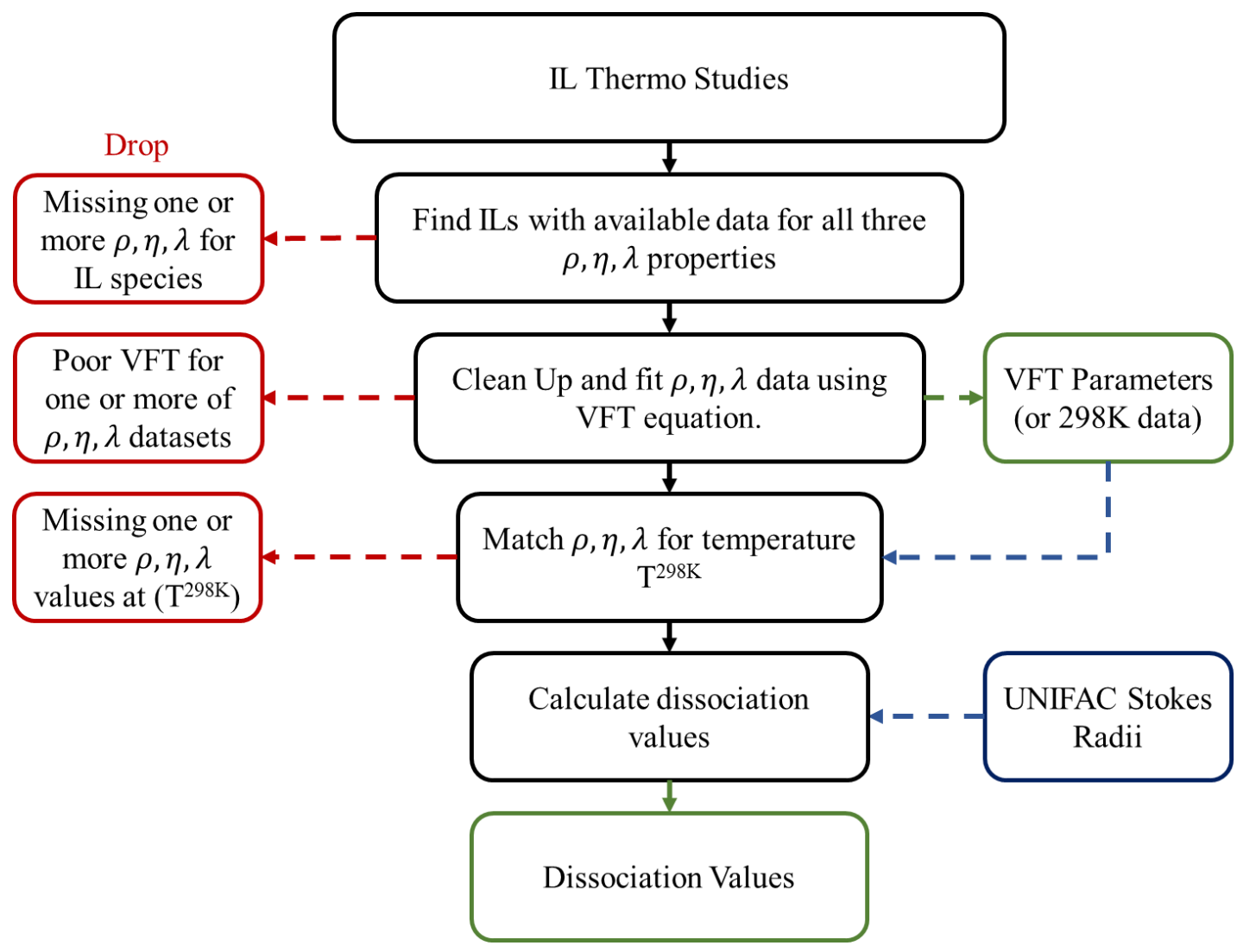

Figure S1. Flowchart of ion dissociation calculations and data analysis for neat IL systems.

To calculate ion dissociation for neat ILs we first compared the available density, viscosity, and ionic conductivity data in the NIST ILThermo database, selecting all ILs for which all three measurements have been reported under the same measurement conditions. It should be noted that in the course of this review only measurements taken under atmospheric pressure were considered. The data for these IL species was then gleaned from the database and checked for consistency across the reported studies. For ILs with multiple studies reporting measurements under identical conditions the data from all studies were compared and outlying studies (studies that differed from the aggregate values by more than $15 \%$ ) were dropped from the final dataset used for the VFT fitting. In the case where only two studies were reported with significantly different values, this IL was then dropped from the final dataset to avoid any selection bias. The framework of these calculations is outlined in Figure S1. 


\section{IL/Solvent Ion Dissociation Calculations:}

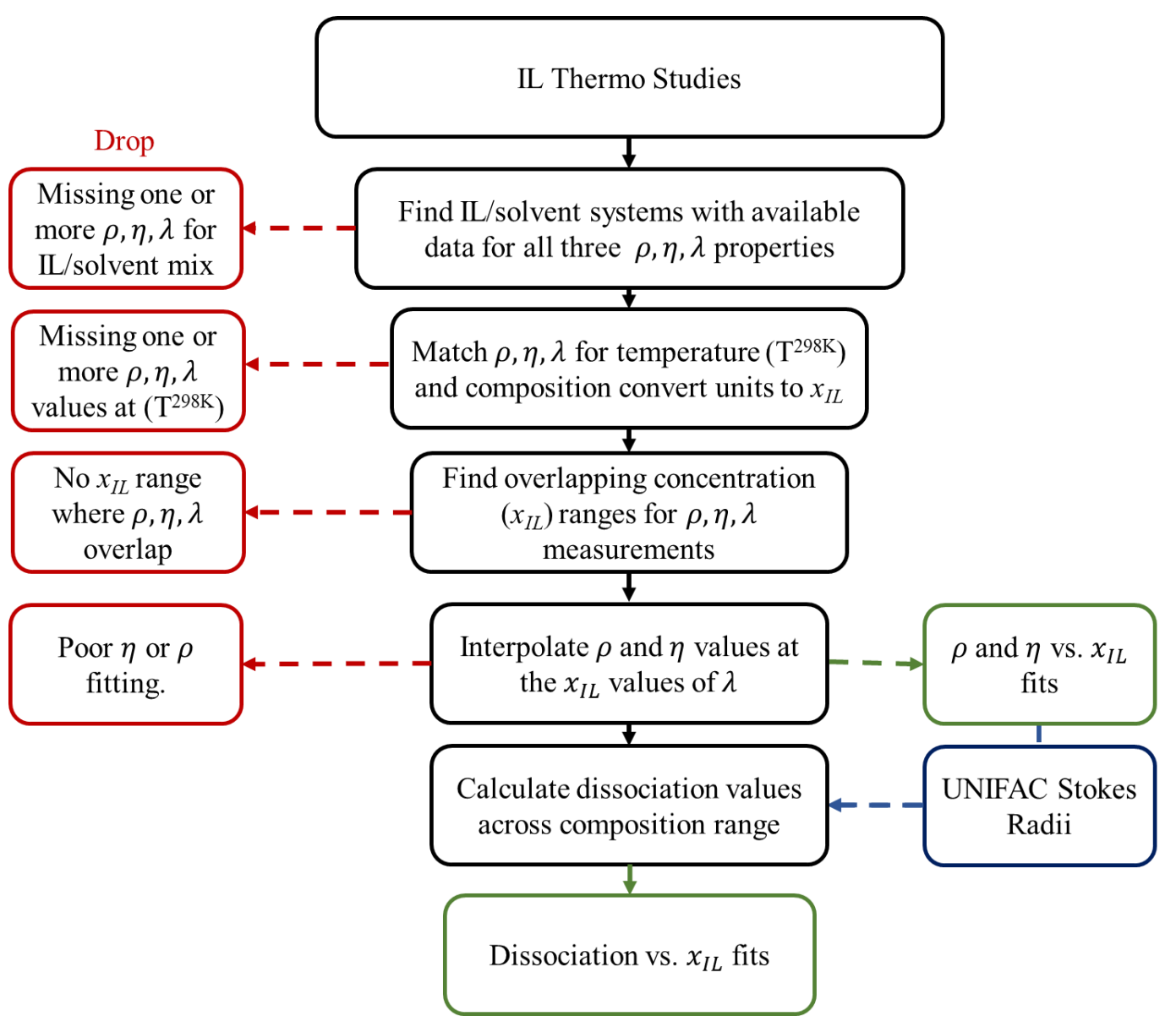

Figure S2. Flowchart of ion dissociation calculations and data analysis for IL/solvent systems.

Ion dissociation calculations for IL/solvent mixtures proved significantly more complex to complete using the reported measurements in the ILThermo Database; however, the general framework followed is similar to that of neat IL systems, as shown in Figure S2. In addition to considering measurements reported at identical temperature and pressure conditions, we also had to account for the IL/solvent compositions of the measurements. Because the units used to report IL/solvent compositions (mole fraction, molality, weight fraction, molarity) varied between studies, all compositions had to be converted to mole fraction prior to further data analysis. Once the respective datasets were translated in terms of property $(\rho, \eta, \kappa)$ vs. $x_{I L}$, the data range for ion dissociation calculations was confined to the composition region $\left(x_{I L}\right)$ where the three property datasets overlapped, or if no such region existed the mixture was removed. This sorting is shown conceptually in Figure S3. 
a.

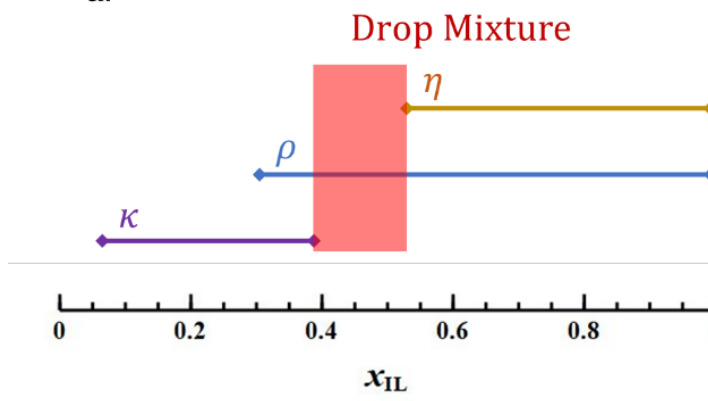

b.

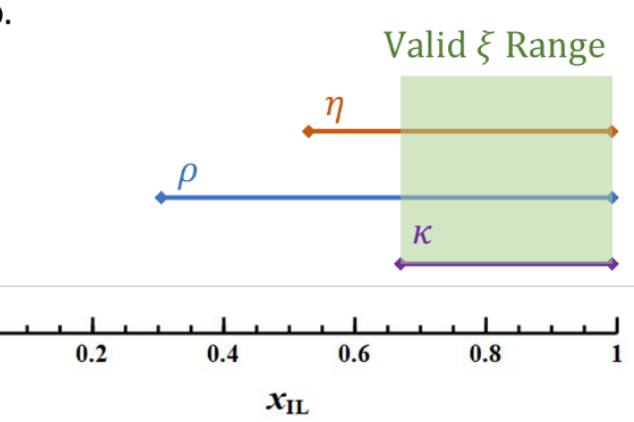

Figure S3. Visual representation of region selection for ILThermo data: a. representation of a mixture with no overlapping data and $\mathbf{b}$. representation of the valid $x_{I L}$ range for ion dissociation calculations.

Once the dissociation calculation composition range was determined, mixtures with fewer than 3 data points were dropped. As discussed in the main manuscript, the density, viscosity and ionic conductivity data is not available at identical compositions for all three properties for most of the systems, requiring fitting of curves (as a function of composition) to two of the three experimental properties. Ionic conductivity displays the most complex composition dependence. Therefore, we use the actual ionic conductivity data points and fit the viscosity and density data (using the Akima1DInterpolator and UnivariateSpline functions, respectively, from scipy.interpolate) as a function of composition to interpolate the density and viscosity values at the same compositions as the ionic conductivity datapoints. While this introduces some additional uncertainty into the ion dissociation calculations, it is an unavoidable step for capturing the change in ion dissociation with composition. An example of these fits is shown in Figure $\mathbf{S} \mathbf{4}$ for 1-butyl-1-methylpyrrolidinium bis(trifluoromethylsulfonyl)imide $\left[\mathrm{C}_{4} \mathrm{mpyrr}\right]\left[\mathrm{Tf}_{2} \mathrm{~N}\right]$ and acetonitrile.
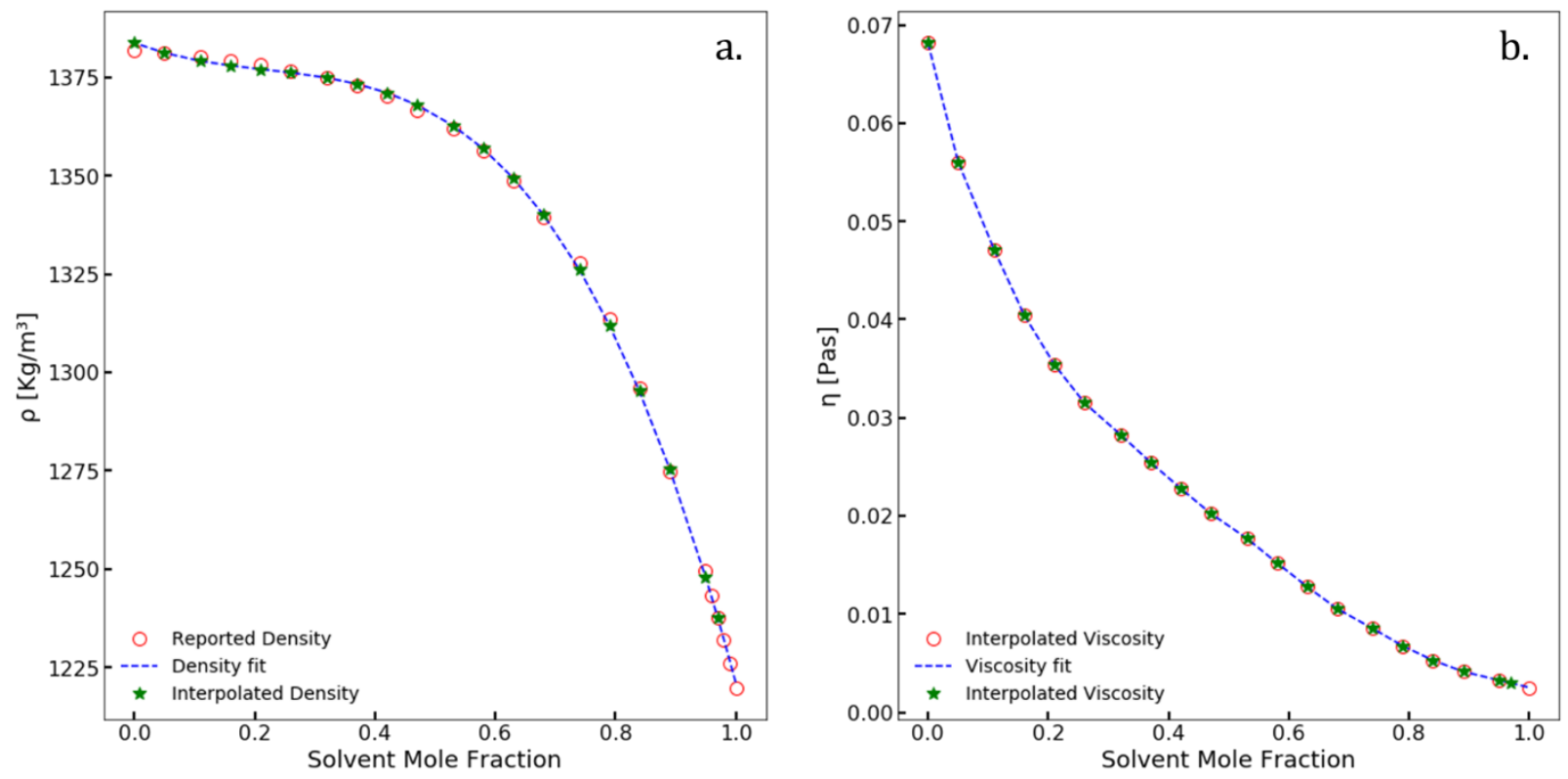

Figure S4. Interpolated densities and viscosities of the $\left[\mathrm{C}_{4} \operatorname{mpyrr}_{[}\right]\left[\mathrm{Tf}_{2} \mathrm{~N}\right]+$ acetonitrile system; the interpolated values are used to calculate the ion dissociation values. 


\section{Vogel-Fulcher-Tammann VFT Fittings:}

As described above, the neat IL ionic conductivity $(\lambda)$ and viscosity $(\eta)$ data were fit using the VogelFulcher-Tammann (VFT) Equation [1,2] (equations S1 and S2) .

$$
\begin{aligned}
& \lambda=A * \exp \left(\frac{-B}{T-C}\right) \\
& \eta=A * \exp \left(\frac{B}{T-C}\right)
\end{aligned}
$$

The respective fit parameters $(\boldsymbol{A}, \boldsymbol{B}, \boldsymbol{C})$ are reported in the supporting information in the

Neat_IL_Supporting_Info.xlsx excel file, as well as the linear fit parameters $(\boldsymbol{a}, \boldsymbol{b})$ for the density $(\rho)$, as shown in equation $\mathbf{S 3}$.

$$
\rho=a-b T
$$

These fits were calculated in python using the scipy.optimize leastsquares library. 